\title{
Classification of reflection matrices related to (super) Yangians and application to open spin chain models
}

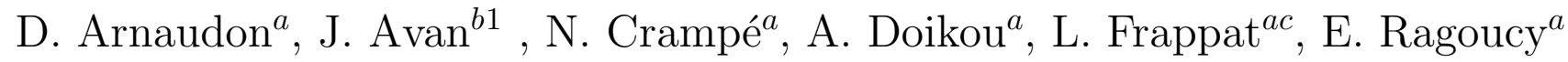 \\ ${ }^{a}$ Laboratoire d'Annecy-le-Vieux de Physique Théorique \\ LAPTH, CNRS, UMR 5108, Université de Savoie \\ B.P. 110, F-74941 Annecy-le-Vieux Cedex, France \\ ${ }^{b}$ Laboratoire de Physique Théorique et Modélisation \\ Université de Cergy, 5 mail Gay-Lussac, Neuville-sur-Oise \\ F-95031 Cergy-Pontoise Cedex \\ ${ }^{c}$ Member of Institut Universitaire de France
}

\begin{abstract}
We present a classification of diagonal, antidiagonal and mixed reflection matrices related to Yangian and super-Yangian $R$ matrices associated to the infinite series $s o(m), s p(n)$ and $\operatorname{osp}(m \mid n)$. We formulate the analytical Bethe Ansatz resolution for the $s o(m)$ and $s p(n)$ open spin chains with boundary conditions described by the diagonal solutions.
\end{abstract}

MSC number: 81R50, 17B37

LAPTH-975/03

math.QA/0304150

March 2003

\footnotetext{
${ }^{1}$ On leave of absence from LPTHE, CNRS, UMR 7589, Universités Paris VI/VII.
} 


\section{Introduction}

Quantum $R$ matrices, solutions of the Yang-Baxter equation, are interpreted as diffusion amplitudes for two-body interactions of particle type eigenstates in integrable two-dimensional field theories. The Yang-Baxter equation then ensures consistent factorisability of the three-body amplitudes in terms of two-body ones.

When considering integrable field theories with non trivial boundary effects such as theories on a half-line, one needs to introduce a new object describing reflection processes on the boundary. Integrability is preserved provided that the two-body exchange $R$ matrix and the one-body reflection $K$ matrix obey a quartic consistency condition [1, 2, 3, 4]

$$
R_{12}(u-v) K_{1}(u) R_{12}(u+v) K_{2}(v)=K_{2}(v) R_{12}(u+v) K_{1}(u) R_{12}(u-v) .
$$

Let us remark that the reflection equation also appears when considering generalisation of ZF algebras (i.e. Zamolodchikov-Faddeev algebras [5]) allowing the presence of a boundary. These generalised $\mathrm{ZF}$ algebras ensure the total scattering matrix of the model to be unitary. In this approach, the commutation relations of ZF generators implement an operator $b(u)$ which obeys the reflection equation. The $K$ matrix would then be a representation of this boundary operator $b[6]$.

Another point of view has recently been presented using a universal construction of reflection algebras as twists of quantum algebras [7, 8]. The exchange equation (1.1) would then describe a trivial representation of the quantum generators, although it is not clear to us that this interpretation is valid for spectral parameter dependent solutions. Furthermore, solutions with quantum degrees of freedom on the boundary have also been obtained in [9].

These reflection equations, or boundary Yang-Baxter equations, have recently drawn attention and systematic ways of computing some solutions for given $R$ matrices were derived e.g. in [10, 11, 12, recovering and extending previous results derived for instance in 13, 14, 15, 16. The considered cases were related to $A_{1}^{(1)}$ trigonometric $R$ matrices for all spins [11], and to $A_{2}^{(2)}$ [17] and $A_{n}^{(1)}$ vector representations [12], yielding a wealth of new solutions.

In this paper, we will consider the case of $R$ matrices corresponding to vector representations of Yangians and super-Yangians, i.e. rational $R$ matrices constructed in [18, 19]. The complete classification for $g l(n)$ in vector representation was given in [20: it appears that generically any solution is conjugated (by a constant matrix) to a diagonal solution. [21] then derived a series of solutions for $s o(m)$ and $s p(n)$ based upon an ansatz proposed by Cherednik [1]. We present here, for the Lie (super)algebra series $s o(m), s p(n)$ and $o s p(m \mid n)$, a classification of purely diagonal, purely antidiagonal and mixed diagonal/antidiagonal solutions by directly solving the boundary equation (1.1) for a one-dimensional boundary quantum space. Once the diagonal case is exhausted (corresponding to "flavour-preserving" reflection matrices), the most natural extension to look for indeed consists of reflection matrices which preserve pairs of conjugate states (according to (2.2)). Particular solutions have already been derived for the $s o(m)$ algebra [21, 22] and the $s p(n)$ algebra [21]. They can all be identified with particular diagonal solutions of our classification.

We must point out that there exists in fact another notion of reflection equation, which is related to the definition of twisted Yangians [23, 24], and arises also in the theory of coideal algebras described 
in 25, 26. This equation reads

$$
R_{12}(u-v) K_{1}(u) R_{12}^{t_{1}}(-u-v) K_{2}(v)=K_{2}(v) R_{12}^{t_{1}}(-u-v) K_{1}(u) R_{12}(u-v)
$$

where the transposition $t$ is defined below (see definition 2.2). As pointed out in [19], this equation is actually the same as (1.1) when $R(u)$ is the $R$ matrix of Yangians of type $s o(n), s p(n)$ or $o s p(m \mid n)$.

One essential purpose in establishing such a classification of reflection matrices is to use them in order to construct and eventually to solve spin chain models with a variety of boundary conditions. We present here explicit resolutions, using the analytical Bethe Ansatz method, of the $s o(n)$ and $s p(n)$ open spin chains with boundary conditions determined by the diagonal solutions of the reflection equations. In particular, we derive explicit formulae for the eigenvalues of the low-lying excitations of hole type. We obtain in each case the full scattering matrix without ambiguities (e.g. CDD factors) including bulk and boundary interactions. This result has in fact a very general character, not limited to the particular models considered here. This actually provides us with bulk and boundary $S$ matrices relevant for integrable field theories with non trivial boundary conditions, for which they represent "universal" $S$ matrices. We expect, in analogy with the bulk case, that such boundary $S$ matrices should correspond to $s p(n)$ or $s o(m)$ Gross-Neveu model with certain boundaries. For this particular model, the study of boundary conditions associated with the different $K$ matrices deserves further attention but goes beyond the scope of the present paper.

\section{Generalities}

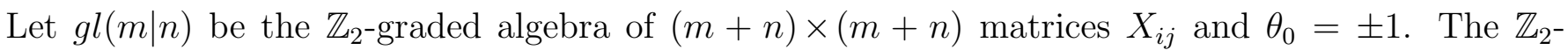
gradation is defined by $(-1)^{[i]}=\theta_{0}$ if $1 \leq i \leq m$ and $(-1)^{[i]}=-\theta_{0}$ if $m+1 \leq i \leq m+n$. In the following, we will always assume that $n$ is even.

Definition 2.1 For each index $i$, we introduce a sign $\theta_{i}$

$$
\theta_{i}=\left\{\begin{array}{lll}
+1 & \text { for } & 1 \leq i \leq m+\frac{n}{2} \\
-1 & \text { for } & m+\frac{n}{2}+1 \leq i \leq m+n
\end{array}\right.
$$

and a conjugate index $\bar{\imath}$

$$
\bar{\imath}= \begin{cases}m+1-i & \text { for } 1 \leq i \leq m \\ 2 m+n+1-i & \text { for } m+1 \leq i \leq m+n\end{cases}
$$

In particular $\theta_{i} \theta_{\bar{\imath}}=\theta_{0}(-1)^{[i]}$.

Definition 2.2 For $A=\sum_{i j} A^{i j} E_{i j}$, we define the transposition $t$ by

$$
A^{t}=\sum_{i j}(-1)^{[i][j]+[j]} \theta_{i} \theta_{j} A^{i j} E_{\bar{\jmath}}=\sum_{i j}\left(A^{i j}\right)^{t} E_{i j}
$$

It satisfies $\left(A^{t}\right)^{t}=A$ and, for $\mathbb{C}$-valued bosonic matrices, $(A B)^{t}=B^{t} A^{t}$. 
As usual $E_{i j}$ denotes the elementary matrix with entry 1 in row $i$ and column $j$ and zero elsewhere. We shall use a graded tensor product, i.e. such that, for $a, b, c$ and $d$ with definite gradings, $(a \otimes b)(c \otimes d)=(-1)^{[b][c]} a c \otimes b d$.

Definition 2.3 Let $P$ be the (super)permutation operator (i.e. $X_{21} \equiv P X_{12} P$ )

$$
P=\sum_{i, j=1}^{m+n}(-1)^{[j]} E_{i j} \otimes E_{j i}
$$

and let

$$
Q=\sum_{i, j=1}^{m+n}(-1)^{[i][j]} \theta_{i} \theta_{j} E_{\bar{\jmath}} \otimes E_{j i}=P^{t_{1}}
$$

We define the $R$ matrix

$$
R(u)=\mathbb{I}+\frac{P}{u}-\frac{Q}{u+\kappa}
$$

with $2 \kappa=(m-n-2) \theta_{0}$.

The $R$ matrix (2.6) satisfies the super Yang-Baxter equation

$$
R_{12}(u) R_{13}(u+v) R_{23}(v)=R_{23}(v) R_{13}(u+v) R_{12}(u)
$$

where the graded tensor product is understood.

The operators $P$ and $Q$ satisfy

$$
P^{2}=\mathbb{I}, \quad P Q=Q P=\theta_{0} Q, \quad \text { and } \quad Q^{2}=\theta_{0}(m-n) Q
$$

The $R$ matrix (2.6]) is known to yield the $\operatorname{osp}(m \mid n)$ Yangian [19], and leads to the non-super orthogonal (taking $\left.n=0, \theta_{0}=1\right)$ and symplectic $\left(m=0, \theta_{0}=-1\right)$ Yangians. For obvious reasons, we will call labels as orthogonal (resp. symplectic), indices $i$ which satisfy $1 \leq i \leq m$ (resp. $m+1 \leq i \leq m+n)$.

Although we will restrict ourselves to the diagonal, antidiagonal and mixed cases for the reflection matrices $K$, the following lemma can be used to get more general solutions.

Lemma 2.4 Let $K(u)$ be a solution of the reflection equation (1.1) and $U$ such that $U U^{t}=1$ be a (constant) matrix of the orthogonal, symplectic or orthosymplectic (super)group (depending upon the choice of $R$ ). Then $K^{t}(u), U K(u) U^{t}$ and $U K^{t}(u) U^{t}$ are also solutions of (1.1).

The proof is straighforward, using the invariance of the $R$ matrix under conjugation by $U$ and $R_{12}^{t_{1} t_{2}}=R_{12}$. 


\section{Diagonal solutions of the reflection equation}

In this section we consider invertible diagonal solutions for $K(u)$, i.e.

$$
K(u)=\operatorname{diag}\left(k_{1}(u), \cdots, k_{m}(u) ; k_{m+1}(u), \cdots, k_{m+n}(u)\right)
$$

where the semicolon emphasises the splitting between orthogonal and symplectic indices. Here the $k_{i}(u)$ are supposed to be analytic $\mathbb{C}$-functions of $u$, i.e. the boundary quantum space is onedimensional.

Proposition 3.1 There are three families of generic diagonal solutions and two particular cases

D1: Solutions of $\operatorname{sl}(m+n)$ type, with one free parameter, for $m$ even

$$
k_{i}(u)=1, \quad k_{\bar{\imath}}(u)=\frac{1+c u}{1-c u}, \quad \forall i \in\left\{1, \ldots, \frac{m}{2} ; m+1, \ldots, m+\frac{n}{2}\right\}
$$

This solution has no extension to odd $m$.

This solution is obviously invariant under the action of $S L\left(\frac{m}{2} \mid \frac{n}{2}\right)$.

D2: Solutions with three different values of $k_{l}(u)$, depending on one free parameter

$$
\begin{aligned}
& k_{1}(u)=\frac{1+c_{1} u}{1-c_{1} u}, \quad k_{m}(u)=\frac{1+c_{m} u}{1-c_{m} u} \\
& k_{j}(u)=1 \quad \forall j \neq 1, m \\
& \text { where } \quad\left(\kappa-\theta_{0}\right) c_{1} c_{m}+c_{1}+c_{m}=0
\end{aligned}
$$

This solution does not hold for $m=0,1$.

The moduli space of this solution is invariant under the action of $O S P(m-2 \mid n) \times S O(2)$.

D3: Solutions without any free continuous parameter (but with two integer parameters)

$$
\begin{aligned}
& k_{i}(u)=k_{\bar{\imath}}(u)=1 \quad \forall i \in\left\{1, \ldots, m_{1} ; m+1, \ldots, m+n_{1}\right\} \\
& k_{i}(u)=k_{\bar{\imath}}(u)=\frac{1+c u}{1-c u} \quad \forall i \in\left\{m_{1}+1, \ldots, m-m_{1} ; m+n_{1}+1, \ldots, m+n-n_{1}\right\} \\
& \text { where } \quad c=\frac{2}{\kappa-\theta_{0}\left(2 m_{1}-2 n_{1}-1\right)}
\end{aligned}
$$

This solution is invariant under the action of $O S P\left(2 m_{1} \mid 2 n_{1}\right) \times O S P\left(m-2 m_{1} \mid n-2 n_{1}\right)$.

D4: In the particular case of so(4), the solution takes the more general form:

$$
K(u)=\operatorname{diag}\left(1, \frac{1+c_{2} u}{1-c_{2} u}, \frac{1+c_{3} u}{1-c_{3} u}, \frac{1+c_{2} u}{1-c_{2} u} \frac{1+c_{3} u}{1-c_{3} u}\right)
$$

This solution contains the three generic solutions D1 $\left(c_{2} c_{3}=0\right)$, D2 $\left(c_{2}+c_{3}=0\right)$ and D3 $\left(c_{2}=c_{3}=\infty\right)$. 
D5: In the particular case of so(2), any function-valued diagonal matrix is solution.

In each case D1-D4, infinite value of the parameter $c$ is allowed. It corresponds to the constant value $\frac{1+c u}{1-c u}=-1$.

These solutions are given up to a global normalisation and a relabelling of the indices, provided it preserves the orthogonal/symplectic splitting and all the sets of conjugate indices $\{i, \bar{\imath}\}$.

Let us remind that the solutions for $s o(m)$ and $s p(n)$ algebras are obtained by setting $n=0$ or $m=0$ respectively.

Solutions found in 22] for $s o(m)$ algebras $(m>2)$ are identified after a suitable change of basis with the sets D1 and D2. Solutions found in [21] for $s o(m)$ and $s p(n)$ algebras are identified with the set D3 and limiting cases of D1. The solution D3 with the special value $m_{1}=(m-1) / 2$ (with $n=0$ ) corresponds to the only non trivial rational limit of the set of trigonometric solutions given in $[28$.

Remark that the generic solutions D1, D2, D3 are associated with symmetric superspaces (as they were introduced in [29], in a different context) based on $O S P(m \mid n)$, namely $O S P(m \mid n) / S L\left(\frac{m}{2} \mid \frac{n}{2}\right)$, $O S P(m \mid n) / O S P(m-2 \mid n) \times S O(2)$, and $O S P(m \mid n) / O S P\left(m-2 m_{1} \mid n-2 n_{1}\right) \times O S P\left(2 m_{1} \mid 2 n_{1}\right)$, respectively. In the case of Lie algebras, we recover the usual symmetric spaces (based on orthogonal and symplectic algebras).

Proof: The projection of the reflection equation (1.1) on $E_{i j} \otimes E_{j i}$ for $i \neq j, \bar{\jmath}$ reads

$$
\frac{1}{u+v} k_{i}(u) k_{i}(v)+\frac{1}{u-v} k_{j}(u) k_{i}(v)=\frac{1}{u+v} k_{j}(u) k_{j}(v)+\frac{1}{u-v} k_{j}(v) k_{i}(u),
$$

the solution of which is given by

$$
\frac{k_{i}(u)}{k_{j}(u)}=\frac{1+c_{i j} u}{1-c_{i j} u}, \quad i \neq j, \bar{\jmath}
$$

The cases $c_{i j}=0, \infty$ correspond to the constant ratios $\frac{k_{i}(u)}{k_{j}(u)}= \pm 1$.

For convenience, we will introduce $F_{i j}(u) \equiv \frac{k_{i}(u)}{k_{j}(u)}$ (well-defined since $K(u)$ is supposed to be invertible for generic $u$ ). When $i \neq j, \bar{\jmath}, F_{i j}$ is then given by (3.7) for some $c_{i j}$ and obviously $c_{j i}=-c_{i j}$. When defined, since $F_{i j}(u) F_{j k}(u)=F_{i k}(u)$, the parameters $c$ must also satisfy

$$
\left\{\begin{array}{l}
c_{i j}+c_{j k}+c_{k i}=0 \\
c_{i j} c_{j k} c_{k i}=0
\end{array} \quad \text { for } \quad i \neq j, \bar{\jmath}, \quad j \neq k, \bar{k} \quad \text { and } k \neq i, \bar{\imath} .\right.
$$

To any solution for $K(u)$, we associate a partition of $\{1, \ldots, m+n\}$ where the classes are defined by $i \equiv j \Leftrightarrow k_{i}=k_{j}\left(\Leftrightarrow c_{i j}=0\right)$. The constraints (3.8) are sufficient to conclude that the partition associated to any solution has at most three different classes, except when $m+n=4$, where it can in principle have four classes. Note that in the case of $\operatorname{sl}(m \mid n)$ where the constraints (3.8) hold without any restriction on the indices, $K(u)$ is always built with at most two different functions.

More precisely, if $m+n \neq 4$, (3.8) implies that the partition of indices is constituted either of two subsets, or of three subsets. In the last case, it can only be of the form 


$$
\{i\},\{\bar{\imath}\} \text { and }\{1, \ldots, m+n\} \backslash\{i, \bar{\imath}\}
$$

where $i$ is an orthogonal index, i.e. $i \in\{1, \ldots, m\}$.

Projecting the reflection equation (1.1) on $E_{\bar{\imath} \bar{\jmath}} \otimes E_{i j}$, one gets (for $i \neq j, \bar{\jmath}$ ) after taking $u \rightarrow v$

$$
\begin{aligned}
\kappa\left(F_{\bar{i} i}(u)-F_{\bar{j} j}(u)\right)+\theta_{0}\left(F_{j i}(u)-F_{i j}(u)\right)+(2 u+\kappa) & \left(F_{\bar{j} i}(u)-F_{\bar{\imath} j}(u)\right) \\
& =\sum_{l}(-1)^{[l]}\left(F_{l i}(u)-F_{l j}(u)\right)
\end{aligned}
$$

This equation show in particular that $F_{i j}=1 \Leftrightarrow F_{\bar{\imath} \bar{\jmath}}=1$. A recursion allows us to write the two-subset partitions as either

$$
I=\left\{i_{1}, i_{2}, \ldots\right\} \text { and } \bar{I}=\left\{\bar{\imath}_{1}, \bar{\imath}_{2}, \ldots\right\} \text {, i.e. } i \in I \Leftrightarrow \bar{\imath} \notin I
$$

or

$$
I=\left\{i_{1}, \bar{\imath}_{1}, i_{2}, \bar{\imath}_{2} \ldots\right\} \text { and } J=\left\{j_{1}, \bar{\jmath}_{1}, j_{2}, \bar{\jmath}_{2}, \ldots\right\} \text {, i.e. } i \in I \Leftrightarrow \bar{\imath} \in I
$$

Knowing the possible forms of the partition, one can evaluate the sum in (3.9). This equation involves at most two different functions $F_{m n}$. It provides the constraints on the parameters $c_{m n}$. A global check ensures that all the remaining projections do not lead to new constraints.

The cases $m+n=4$ are solved by direct computation. Only the so(4) case eventually exhibits a more general solution (D4).

Finally, the case of $s o(2)$ is special. In particular, $R(u)$ appears to be diagonal. A direct computation shows that all function-valued diagonal matrix is solution.

Note that when $\kappa=0$ (as in the case of $s o(2)$ ), the spectral parameter can be rescaled before taking the limit $\kappa \rightarrow 0$, and the corresponding $R$ matrix does not involve the identity anymore 30]. This is the $R$ matrix used in [22] for $s o(2)$.

\section{Antidiagonal solutions of the reflection equation}

We now look for invertible solutions of the "antidiagonal" form

$$
K(u)=\sum_{i=1}^{m+n} \ell_{i}(u) E_{i \bar{\imath}} .
$$

Proposition 4.1 Solutions of the reflection equation with antidiagonal terms exist only in the pure so $(2 m)$ or $s p(2 n)$ cases. They are constant and only restricted by the set of constraints

$$
\ell_{i} \ell_{\bar{\imath}}=1, \quad \forall i
$$

In the special case of so(2), any function-valued antidiagonal matrix is solution.

The proof of this proposition will be given in the next section since the antidiagonal solutions appear to be particular cases of the mixed solutions discussed below. 


\section{Mixed solutions of the reflection equation}

We now look for invertible solutions with terms both in the diagonal and in the antidiagonal part

$$
K(u)=\sum_{i=1}^{m+n}\left(k_{i}(u) E_{i i}+\ell_{i}(u) E_{i \bar{\imath}}\right)
$$

with at least one non zero $\ell_{i}$.

Lemma 5.1 The case of so(2) being excluded, the solutions of the reflection equation of the form (5.1) are all constant (up to a global normalisation function).

In the special case of so(2), the set of solutions of the reflection equation is the union of functionvalued diagonal matrices and function-valued antidiagonal matrices.

Proof: Projecting the reflection equations on the elementary matrices $E_{p q} \otimes E_{r s}$, one gets

$$
\begin{array}{ll}
\text { on } E_{i j} \otimes E_{j \bar{\imath}}: & \frac{(-1)^{[j]}}{u+v}\left(k_{i}(u) \ell_{i}(v)+k_{\bar{\imath}}(v) \ell_{i}(u)\right)+\frac{(-1)^{[j]}}{u-v}\left(k_{j}(u) \ell_{i}(v)-k_{j}(v) \ell_{i}(u)\right)=0 \\
\text { on } E_{i j} \otimes E_{\bar{\jmath} i}: & -\frac{(-1)^{[j]}}{u+v}\left(k_{j}(u) \ell_{\bar{\jmath}}(v)+k_{\bar{\jmath}}(v) \ell_{\bar{\jmath}}(u)\right)-\frac{(-1)^{[j]}}{u-v}\left(k_{i}(u) \ell_{\bar{\jmath}}(v)-k_{i}(v) \ell_{\bar{\jmath}}(u)\right)=0
\end{array}
$$

on $E_{i j} \otimes E_{j i}: \quad \frac{(-1)^{[j]}}{u+v}\left(k_{i}(u) k_{i}(v)+\ell_{i}(u) \ell_{\bar{\imath}}(v)-k_{j}(u) k_{j}(v)-\ell_{\bar{\jmath}}(u) \ell_{j}(v)\right)$

$$
-\frac{(-1)^{[j]}}{u-v}\left(k_{i}(u) k_{j}(v)-k_{j}(u) k_{i}(v)\right)=0
$$

on $E_{i j} \otimes E_{\bar{\jmath}}: \quad-\frac{(-1)^{[j]}}{u-v}\left(\ell_{i}(u) \ell_{\bar{\jmath}}(v)-\ell_{i}(v) \ell_{\bar{\jmath}}(u)\right)=0$

on $E_{i j} \otimes E_{i j}: \quad-\frac{(-1)^{[i][j]+[i]+[j]}}{u+v+\kappa} \theta_{i} \theta_{j} \theta_{0}\left\{\left((-1)^{[i]} \ell_{i}(u) \ell_{\bar{\jmath}}(v)-(-1)^{[j]} \ell_{\bar{\jmath}}(u) \ell_{i}(v)\right)\right.$

$$
\left.+\frac{1}{u-v}\left(\ell_{i}(u) \ell_{\bar{\jmath}}(v)-\ell_{\bar{\jmath}}(u) \ell_{i}(v)\right)\right\}=0
$$

on $E_{i j} \otimes E_{\bar{\imath} \bar{\jmath}}: \quad(-1)^{[i][j]+[i]+[j]} \theta_{i} \theta_{j}\left\{\frac{-1}{u+v+\kappa}\left(k_{i}(u) k_{\bar{\jmath}}(v)-k_{j}(u) k_{\bar{\imath}}(v)\right)+\right.$

$$
\begin{aligned}
& +\frac{1}{u-v+\kappa}\left(k_{i}(u) k_{\bar{\imath}}(v)-k_{j}(u) k_{\bar{\jmath}}(v)+(-1)^{[i]} \ell_{i}(u) \ell_{\bar{\imath}}(v)-(-1)^{[j]} \ell_{\bar{\jmath}}(u) \ell_{j}(v)\right) \\
& -\frac{1}{u+v+\kappa}\left(\frac{\theta_{0}}{u-v}\left(k_{\bar{\imath}}(u) k_{\bar{\jmath}}(v)-k_{\bar{\jmath}}(u) k_{\bar{\imath}}(v)\right)+\frac{\operatorname{Str} K(u)}{u-v+\kappa}\left(k_{\bar{\imath}}(v)-k_{\bar{\jmath}}(v)\right)\right) \\
& \left.+\frac{\theta_{0}}{(u+v)(u-v+\kappa)}\left(k_{\bar{\imath}}(u) k_{\bar{\imath}}(v)-k_{\bar{\jmath}}(u) k_{\bar{\jmath}}(v)+\ell_{i}(u) \ell_{\bar{\imath}}(v)-\ell_{\bar{\jmath}}(u) \ell_{j}(v)\right)\right\}=0
\end{aligned}
$$




$$
\text { on } \begin{aligned}
E_{i j} \otimes E_{\bar{\imath} j}: \quad & (-1)^{[i][j]+[i]+[j]} \theta_{i} \theta_{j}\left\{\frac{-1}{u+v+\kappa}\left(k_{i}(u) \ell_{\bar{\jmath}}(v)-(-1)^{[j]} \theta_{0} \ell_{\bar{\jmath}}(u) k_{\bar{\imath}}(v)\right)+\right. \\
& -\frac{1}{u-v+\kappa}\left(k_{j}(u) \ell_{\bar{\jmath}}(v)+(-1)^{[j]} \theta_{0} \ell_{\bar{\jmath}}(u) k_{j}(v)+\frac{\theta_{0}}{u+v}\left(k_{\bar{\jmath}}(u) \ell_{\bar{\jmath}}(v)+\ell_{\bar{\jmath}}(u) k_{j}(v)\right)\right) \\
& \left.-\frac{1}{u+v+\kappa}\left(\frac{\theta_{0}}{u-v}\left(k_{\bar{\imath}}(u) \ell_{\bar{\jmath}}(v)-\ell_{\bar{\jmath}}(u) k_{\bar{\imath}}(v)\right)-\frac{\operatorname{Str} K(u)}{u-v+\kappa} \ell_{\bar{\jmath}}(v)\right)\right\}=0
\end{aligned}
$$

on $E_{i j} \otimes E_{i \bar{\jmath}}: \quad(-1)^{[i][j]+[i]+[j]} \theta_{i} \theta_{j}\left\{\frac{1}{u+v+\kappa}\left(k_{j}(u) \ell_{i}(v)-(-1)^{[i]} \theta_{0} \ell_{i}(u) k_{\bar{\jmath}}(v)\right)+\right.$

$$
\begin{aligned}
& +\frac{1}{u-v+\kappa}\left(k_{i}(u) \ell_{i}(v)+(-1)^{[i]} \theta_{0} \ell_{i}(u) k_{i}(v)+\frac{\theta_{0}}{u+v}\left(k_{\bar{\imath}}(u) \ell_{i}(v)+\ell_{i}(u) k_{i}(v)\right)\right) \\
& \left.-\frac{1}{u+v+\kappa}\left(\frac{\theta_{0}}{u-v}\left(\ell_{i}(u) k_{\bar{\jmath}}(v)-k_{\bar{\jmath}}(u) \ell_{i}(v)\right)+\frac{\operatorname{Str} K(u)}{u-v+\kappa} \ell_{i}(v)\right)\right\}=0
\end{aligned}
$$

on $1 \otimes E_{i i}: \quad \frac{1}{u^{2}-v^{2}}\left(\ell_{i}(u) \ell_{\bar{\imath}}(v)-\ell_{\bar{\imath}}(u) \ell_{i}(v)\right)=0$

on $1 \otimes E_{i \bar{\imath}}: \quad \frac{1}{u^{2}-v^{2}}\left(k_{i}(u) \ell_{i}(v)+\ell_{i}(u) k_{\bar{\imath}}(v)-k_{\bar{\imath}}(u) \ell_{i}(v)-\ell_{i}(u) k_{i}(v)\right)=0$

Of course, when several indices $i, j, \bar{\imath}, \bar{\jmath}$ coincide, the corresponding equations merge into a single one. Consider now a couple $(i, j)$ of indices such that $i, j, \bar{\imath}, \bar{\jmath}$ are all different. Then eq. (5.5) implies

$$
\ell_{i}(u) \ell_{\bar{\jmath}}(v)=\ell_{\bar{\jmath}}(u) \ell_{i}(v)
$$

the solution of which is given by $\ell_{i}(u)=\ell_{i}(0) \ell(u)$ where $\ell(u)$ is an arbitrary function, which can be factorised by a change of normalisation of the diagonal elements. Hence one can restrict $K$ in (5.1) to have only constant antidiagonal elements without loss of generality.

Suppose now that $\ell_{i} \neq 0$ for some $i$ (purely diagonal solutions are not considered in this section). The general solution to the system formed by eqs. (15.2) and (5.11) is then given by ${ }^{2}$

$$
k_{i}(u)=\gamma u+k_{i}(0) \quad ; \quad k_{\bar{\imath}}(u)=\gamma u-k_{i}(0) \quad \text { and } \quad k_{j}(u)=-\gamma u+k_{j}(0) \quad(j \neq i, \bar{\imath})
$$

where $\gamma$ is a constant. The inspection of the $E_{\bar{\imath}} \otimes E_{i \bar{\imath}}$ coefficient leads to $\gamma=0$.

It follows that all diagonal terms are constant. Hence the mixed solutions for the reflection matrix are necessarily constant.

The case of $s o(2)$ is solved by direct calculation.

We now specify the exact form of these solutions. We establish (the case of $s o(2)$ being excluded):

Proposition 5.2 Mixed solutions of the reflection equation for osp $(m \mid n)$ exist only when $m$ is even. They fall into two classes:

\section{C1: The so(m) block is diagonal.}

\footnotetext{
${ }^{2}$ Actually, we have restricted ourselves to meromorphic functions on $\mathbb{C}$.
} 
The solutions are parametrised by $n$ complex parameters. The matrix $K$ is given by:

$$
\begin{aligned}
\text { for } i \in\left\{1, \ldots, \frac{m}{2}\right\}: & \begin{cases}k_{i}=1 & k_{\bar{\imath}}=-1 \\
\ell_{i}=0 & \ell_{\bar{\imath}}=0\end{cases} \\
\text { for } i \in\{m+1, \ldots, m+n\}: & \left\{\begin{array}{ll}
k_{i}=\sin \left(\alpha_{i}\right) \\
\ell_{i}=e^{\beta_{i}} \cos \left(\alpha_{i}\right)
\end{array} \text { with } \begin{array}{l}
\alpha_{i}+\alpha_{\bar{\imath}}=0 \\
\beta_{i}+\beta_{\bar{\imath}}=0
\end{array}\right.
\end{aligned}
$$

C2: The $\operatorname{sp}(n)$ block is diagonal.

The solutions are parametrised by a couple of positive or null integers $m_{1} \geq m_{2}$ such that

$$
m_{1}+m_{2} \leq \frac{m}{2}-1 ; \quad m_{1}-m_{2} \leq \frac{n}{2} \quad \text { and } \quad m_{1}-m_{2} \equiv \frac{n}{2}[\bmod 2]
$$

and by $m-2\left(m_{1}+m_{2}\right)$ complex parameters. Setting $n_{1}=\left(n+2 m_{1}-2 m_{2}\right) / 4$, the matrix $K$ is given by:

$$
\begin{aligned}
& \text { so }(m) \text { part: } \begin{cases}\text { for } i \in\left\{1, \ldots, m_{1}\right\}: & {\left[\begin{array}{l}
k_{i}=k_{\bar{\imath}}=1 \\
\ell_{i}=\ell_{\bar{\imath}}=0
\end{array}\right.} \\
\text { for } i \in\left\{m_{1}+1, \ldots, m_{1}+m_{2}\right\}: & {\left[\begin{array}{l}
k_{i}=k_{\bar{\imath}}=-1 \\
\ell_{i}=\ell_{\bar{\imath}}=0
\end{array}\right.} \\
\text { for } i \in\left\{m_{1}+m_{2}+1, \ldots, \frac{m}{2}\right\}: & {\left[\begin{array}{l}
k_{i}=k_{\bar{\imath}}=0 \\
\ell_{\bar{\imath}}=\ell_{i}^{-1}, \ell_{i} \in \mathbb{C}
\end{array}\right.}\end{cases} \\
& \operatorname{sp}(n) \text { part }: \begin{cases}\text { for } i \in\left\{m+1, \ldots, m+n_{1}\right\}: & {\left[\begin{array}{l}
k_{i}=k_{\bar{\imath}}=1 \\
\ell_{i}=\ell_{\bar{\imath}}=0
\end{array}\right.} \\
\text { for } i \in\left\{m+n_{1}+1, \ldots, m+\frac{n}{2}\right\}: & {\left[\begin{array}{l}
k_{i}=k_{\bar{\imath}}=-1 \\
\ell_{i}=\ell_{\bar{\imath}}=0
\end{array}\right.}\end{cases}
\end{aligned}
$$

For so(m) (resp. sp(n)), the mixed solutions are of the form C2 with $n=0$ and $m_{2}=m_{1}$ (resp. C1 with $m=0)$.

Note that this classification is given up to a global normalisation function and up to a relabelling of the indices, as noticed in proposition 3.1 .

Proof: We have to consider only constant reflection matrices $K$. Then, extracting the residues at the poles in $u$, eqs. (5.2) to (5.11) reduce to (for $i \neq \bar{\imath}, j, \bar{\jmath}$ and $j \neq \bar{\jmath}$ )

$$
\begin{aligned}
& \ell_{i}\left(k_{i}+k_{\bar{\imath}}\right)=0 \\
& k_{i}^{2}-k_{j}^{2}+\ell_{i} \ell_{\bar{\imath}}-\ell_{j} \ell_{\bar{\jmath}}=0 \\
& k_{i} k_{\bar{\imath}}-k_{j} k_{\bar{\jmath}}+\theta_{0}\left((-1)^{[i]} \ell_{i} \ell_{\bar{\imath}}-(-1)^{[j]} \ell_{j} \ell_{\bar{\jmath}}\right)=0 \\
& \left((-1)^{[j]}-(-1)^{[i]}\right) \ell_{i} \ell_{j}=0 \\
& k_{i} k_{\bar{\jmath}}-k_{j} k_{\bar{\imath}}=0 \\
& \left(k_{\bar{\imath}}-(-1)^{[j]} \theta_{0} k_{i}\right) \ell_{j}=0 \\
& \left(1+(-1)^{[i]} \theta_{0}\right) k_{i} \ell_{i}=0 \\
& \left(1+(-1)^{[i]} \theta_{0}\right) k_{i} \ell_{\bar{\imath}}=0 \\
& \left(k_{i}-k_{j}\right) \operatorname{Str} K=0 \\
& \ell_{j} \operatorname{Str} K=0
\end{aligned}
$$


In the case of $s o(m)$ algebras, eq. (5.22) implies $k_{i}=k_{\bar{\imath}}$ for all $1 \leq i \leq m$, from which (5.21) follows. Eq. (5.17) is a consequence of (5.23) and (5.24), eq. (5.25) follows from (5.26) and (5.19) from (5.18). Finally the mixed solutions in the $s o(m)$ case are characterised by the following equations:

$$
\begin{array}{ll}
k_{i}=k_{\bar{\imath}} ; \quad k_{i} \ell_{i}=0 ; & \operatorname{Tr} K=0 \\
k_{i}^{2}+\ell_{i} \ell_{\bar{\imath}}=k_{j}^{2}+\ell_{j} \ell_{\bar{\jmath}} & \text { for all } i, j
\end{array}
$$

These constraints exclude the existence of mixed or antidiagonal solutions with odd $m$, since a matrix cannot be traceless if its diagonal has an odd number of non vanishing elements with equal squares.

In the case of $s p(n)$ algebras, eqs. (5.23) and (5.24) vanish, while eq. (5.22) implies $k_{i}=-k_{\bar{\imath}}$ for all $1 \leq i \leq n$, from which (5.17), (5.21), (5.25) and (5.26) follow. Moreover, eqs. (5.18) and (5.19) are equal. Finally the mixed solutions in the $s p(n)$ case are characterised by the following equations:

$$
\begin{aligned}
& k_{i}=-k_{\bar{\imath}} ; \quad \operatorname{Tr} K=0 \\
& k_{i}^{2}+\ell_{i} \ell_{\bar{\imath}}=k_{j}^{2}+\ell_{j} \ell_{\bar{\jmath}} \quad \text { for all } i, j
\end{aligned}
$$

In the case of $\operatorname{osp}(1 \mid n)$, one has to add the following equations (with $2 \leq i, \bar{\imath} \leq 1+n$ )

$$
\begin{aligned}
& k_{1}^{2}-k_{i}^{2}-\ell_{i} \ell_{\bar{\imath}}=0 \\
& k_{1}^{2}-k_{i} k_{\bar{\imath}}-\ell_{i} \ell_{\bar{\imath}}=0
\end{aligned}
$$

It follows from (5.31) -(5.32) and (5.24) that $k_{i}^{2}=0$ for all $i=2, \ldots, 1+n$. Since $\ell_{i} \operatorname{Str} K=0$, one has also $k_{1}=0$. Therefore, there does not exist any mixed or antidiagonal (invertible) solution for $\operatorname{osp}(1 \mid n)$.

In the case of $\operatorname{osp}(m \mid n),(m>1)$, eq. (5.20) shows that at least one antidiagonal part (orthogonal or symplectic) is zero: $\ell_{i}=0$ for $i \in[1, m]$ or $\ell_{i}=0$ for $i \in[m+1, m+n]$. This means that no pure antidiagonal solution exists for $\operatorname{osp}(m \mid n)$ superalgebras. We thus have to consider two cases.

- Case 1 : the $s o(m)$ block is diagonal

Eq. (5.22) with index $j \in[m+1, m+n]$ implies $k_{i}=-k_{\bar{\imath}}$ for all $i$, which excludes the case of $o s p(m \mid n)$ with odd $m$. The remaining equations then lead to

$$
\begin{aligned}
& k_{i}^{2}+\ell_{i} \ell_{\bar{\imath}}=k_{j}^{2}+\ell_{j} \ell_{\bar{\jmath}} \quad \text { for } i, j \in[1, m+n] \\
& k_{j}=-k_{\bar{\jmath}} \quad \text { for } j \in[1, m+n]
\end{aligned}
$$

- Case 2 : the $s p(n)$ block is diagonal

Eq. (5.22) with index $j \in[1, m]$ implies $k_{i}=k_{\bar{\imath}}$ for all $i$. The remaining equations lead then to

$$
\begin{aligned}
& k_{i}^{2}+\ell_{i} \ell_{\bar{\imath}}=k_{j}^{2}+\ell_{j} \ell_{\bar{\jmath}} \quad \text { for } i, j \in[1, m+n] \\
& k_{j}=k_{\bar{\jmath}} \quad \text { for } j \in[1, m+n] \\
& k_{i} \ell_{i}=k_{i} \ell_{\bar{\imath}}=0 \quad \text { for } i \in[1, m] \\
& \operatorname{Str} K=\left(\sum_{i=1}^{m}-\sum_{i=m+1}^{m+n}\right) k_{i}=0
\end{aligned}
$$


Again, these constraints exclude the existence of mixed or antidiagonal solutions with odd $m$, since one cannot have a traceless matrix if its diagonal has an odd number of non vanishing elements with equal squares.

For example, one finds the two following solutions for $\operatorname{ssp}(4 \mid 2)$ :

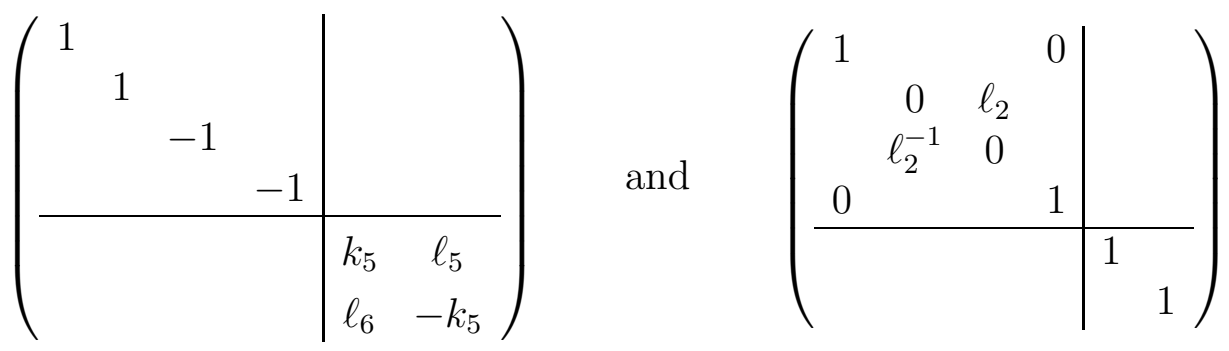

where $k_{5}^{2}+\ell_{5} \ell_{6}=1$. For $\operatorname{osp}(2 \mid 4)$ the two solutions take the form

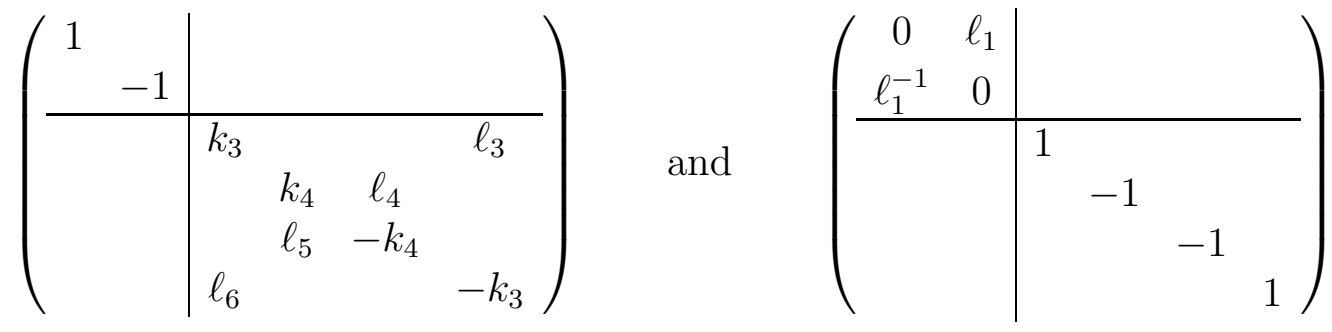

where $k_{3}^{2}+\ell_{3} \ell_{6}=1$ and $k_{4}^{2}+\ell_{4} \ell_{5}=1$.

\section{Analytical Bethe Ansatz for the $\operatorname{so}(n)$ and $\operatorname{sp}(n)$ open spin chains}

The main aim of this section is the derivation of the Bethe Ansatz equations for the $s o(n)$ and $s p(n) N$-site open spin chains with non trivial reflection conditions by means of the analytical Bethe Ansatz method (see e.g. [31, 32, 33, 34, 35, 36]). To construct the open chain transfer matrix we need to introduce the $R$ matrix [37, 38, which is a solution of the Yang-Baxter equation (2.7). Normalisation of $R$ matrix will be modified in order to connect it with the physical $X X X$ type Hamiltonians considered hereafter; in addition, we set $\lambda=i u$. We focus on the $\operatorname{so}(n)$ or $\operatorname{sp}(n)$ invariant $R$ matrix given by:

$$
R(\lambda)=\lambda(\lambda+i \kappa) \mathbb{I}+i(\lambda+i \kappa) P-i \lambda Q .
$$

The $R$ matrix (6.1) satisfies also crossing and unitarity, namely

$$
R_{12}(\lambda) R_{12}(-\lambda)=\left(\lambda^{2}+\kappa^{2}\right)\left(\lambda^{2}+1\right) \mathbb{I}, \quad R_{12}(\lambda)=R_{12}^{t_{1}}(-\lambda-i \kappa) .
$$

where ${ }^{t}$ is the transposition defined as in (2.3).

The open chain transfer matrix is defined by [2]

$$
t(\lambda)=\operatorname{Tr}_{0} K_{0}^{+}(\lambda) T_{0}(\lambda) K_{0}^{-}(\lambda) \hat{T}_{0}(\lambda)
$$


where $K_{0}^{-}(\lambda)$ is any solution of eq. (1.1) and $K_{0}^{+}(\lambda)$ is a solution of a closely related reflection equation defined to be:

$$
R_{12}(v-u) K_{1}^{t_{1}}(u) R_{12}(-u-v-2 i \kappa) K_{2}^{t_{2}}(v)=K_{2}^{t_{2}}(v) R_{12}(-u-v-2 i \kappa) K_{1}^{t_{1}}(u) R_{12}(v-u)
$$

$\operatorname{Tr}_{0}$ denotes trace over the auxiliary space 0 , and

$$
T_{0}(\lambda)=R_{0 N}(\lambda) R_{0 N-1}(\lambda) \cdots R_{02}(\lambda) R_{01}(\lambda), \quad \hat{T}_{0}(\lambda)=R_{10}(\lambda) R_{20}(\lambda) \cdots R_{N-10}(\lambda) R_{N 0}(\lambda)
$$

It is clear that any solution $K^{-}\left(\lambda, \xi^{-}\right)$of (1.1) where $\xi^{-}$are arbitrary boundary parameters, give rise to a solution $K^{+}(\lambda)$ of (6.4) defined by $K^{+}(\lambda)=K^{-}(-\lambda-i \kappa)^{t}$, also depending on arbitrary boundary parameters $\xi^{+}=\xi^{-}$.

To determine the eigenvalues of the transfer matrix and the corresponding Bethe Ansatz equations, we employ the analytical Bethe Ansatz method [31, 32. Namely, we impose certain constraints on the eigenvalues by exploiting the crossing symmetry of the model, the symmetry of the transfer matrix, the analyticity of the eigenvalues, and the fusion procedure for open spin chains. This then allows us to determine the eigenvalues by solving a set of coupled non-linear consistency equations or Bethe Ansatz equations.

We first focus on the case with trivial boundaries, namely $K^{-}(\lambda)=K^{+}(\lambda)=1$. We will in a second step derive the Bethe Ansatz equations for all diagonal solutions found in the previous sections, namely D1, D2, D3, D4 and solve them in the thermodynamical limit.

To obtain the necessary constraints, we recall that the fusion procedure for the open spin chain [39, 40, 36] yields the fused transfer matrix

$$
\tilde{t}(\lambda)=\zeta(2 \lambda+2 i \kappa) t(\lambda) t(\lambda+i \kappa)-(\zeta(\lambda+i \kappa))^{2 N} q(2 \lambda+i \kappa) q(-2 \lambda-3 i \kappa),
$$

where we define

$$
\begin{aligned}
& \zeta(\lambda)=(\lambda+i \kappa)(\lambda+i)(\lambda-i \kappa)(\lambda-i),
\end{aligned}
$$

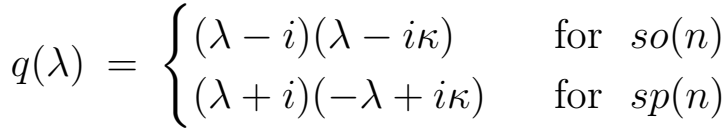

In addition, from the crossing symmetry of the $R$ matrix (6.2) it follows that (when $K^{-}=K^{+}=1$ ):

$$
t(\lambda)=t(-\lambda-i \kappa)
$$

The transfer matrix with $K^{-}=K^{+}=1$ is obviously $s o(n)$ (resp. $s p(n)$ ) invariant since the corresponding $R$ matrix (6.1) is $s o(n)$ (resp. $s p(n)$ ) invariant. The symmetry of the transfer matrix makes the computation of its asymptotic behaviour $(\lambda \rightarrow \infty)$ a relatively easy task. Finally, to implement the analyticity of the eigenvalues, we require that all poles must vanish. These constraints shall uniquely fix the eigenvalues. This is the basic outline of the analytical Bethe Ansatz method. 


\subsection{Eigenvalue of the pseudo-vacuum}

In order to compute the general eigenvalues we need to first define a reference state or "pseudovacuum". After finding its pseudo-energy eigenvalue, we will be able, with the help of the above discussed constraints, to derive the general eigenvalues of the transfer matrix. The pseudo-vacuum, which is an exact eigenstate of the transfer matrix, is the state with all "spins" up, i.e.

$$
\left|\omega_{+}\right\rangle=\bigotimes_{i=1}^{N}|+\rangle_{i}
$$

where $|+\rangle$ is the $n$-dimensional column vector

$$
|+\rangle=\left(\begin{array}{c}
1 \\
0 \\
\vdots \\
0
\end{array}\right)
$$

The action of the $R$ matrix on the state $|+\rangle$ from the left gives rise to upper triangular matrices, whereas the action from the right on $\langle+|=|+\rangle^{\dagger}$ gives lower triangular matrices. It is obvious then that the corresponding action of the $\hat{T}$ (resp. $T$ ) on $\left|\omega_{+}\right\rangle$(resp. $\left.\left\langle\omega_{+}\right|\right)$will also give rise to upper (resp. lower) triangular matrices. It is relatively easy, after some tedious algebra (see 36] for a detailed example), to determine the action of the transfer matrix, $t(\lambda)\left|\omega_{+}\right\rangle=\Lambda^{0}(\lambda)\left|\omega_{+}\right\rangle$, where $\Lambda^{0}(\lambda)$ is given by the following expression

$$
\Lambda^{0}(\lambda)=a(\lambda)^{2 N} g_{0}(\lambda)+b(\lambda)^{2 N} \sum_{l=1}^{n-2} g_{l}(\lambda)+c(\lambda)^{2 N} g_{n-1}(\lambda)
$$

with

$$
\begin{aligned}
& a(\lambda)=(\lambda+i)(\lambda+i \kappa), \quad b(\lambda)=\lambda(\lambda+i \kappa), \quad c(\lambda)=\lambda(\lambda+i \kappa-i)=a(-\lambda-i \kappa) \\
& g_{0}(\lambda)=\frac{\left(\lambda+\frac{i \kappa}{2} \pm \frac{i}{2}\right)(\lambda+i \kappa)}{\left(\lambda+\frac{i \kappa}{2}\right)\left(\lambda+\frac{i}{2}\right)}, \quad g_{n-1}(\lambda)=\frac{\left(\lambda+\frac{i \kappa}{2} \mp \frac{i}{2}\right) \lambda}{\left(\lambda+\frac{i \kappa}{2}\right)\left(\lambda+i \kappa-\frac{i}{2}\right)}=g_{0}(-\lambda-i \kappa) .
\end{aligned}
$$

In $g_{0}$ and $g_{n-1}$ the upper sign corresponds to $s o(n)$ and the lower sign to $\operatorname{sp}(n)$ ). Moreover (when $n=2 k)$,

$$
\begin{aligned}
& g_{l}(\lambda)=\frac{\lambda\left(\lambda+\frac{i \kappa}{2} \pm \frac{i}{2}\right)(\lambda+i \kappa)}{\left(\lambda+\frac{i \kappa}{2}\right)\left(\lambda+\frac{i l}{2}\right)\left(\lambda+\frac{i(l+1)}{2}\right)}, \quad 0<l<k, \\
& g_{l}(\lambda)=g_{n-l-1}(-\lambda-i \kappa), \quad k \leq l<n-1
\end{aligned}
$$

This expression is valid for $s o(2 k)$ with the upper sign and for $s p(2 k)$ with the lower sign. For the $s o(2 k+1)$ chain the expressions for $g_{l}, l \neq k$, are the same as in the $s o(2 k)$ chain, except for

$$
g_{k}(\lambda)=\frac{\lambda(\lambda+i \kappa)}{\left(\lambda+\frac{i k}{2}\right)\left(\lambda+\frac{i(k-1)}{2}\right)}=g_{k}(-\lambda-i \kappa) .
$$




\subsection{Dressing functions}

Now that we have the expression for the pseudo-vacuum eigenvalue, in accordance with the general analytical Bethe Ansatz procedure, we make the following assumption for the structure of the general eigenvalues:

$$
\Lambda(\lambda)=a(\lambda)^{2 N} g_{0}(\lambda) A_{0}(\lambda)+b(\lambda)^{2 N} \sum_{i=1}^{n-2} g_{i}(\lambda) A_{i}(\lambda)+c(\lambda)^{2 N} g_{n-1}(\lambda) A_{n-1}(\lambda)
$$

where $A_{i}(\lambda)$, the so-called "dressing functions", will now be determined.

We immediately get from the crossing symmetry of the transfer matrix (6.9):

$$
A_{l}(\lambda)=A_{n-l-1}(-\lambda-i \kappa) \quad 0 \leq l \leq n-1
$$

Moreover, from the fusion relation (6.6), we obtain the following identity by a comparison of the forms (6.17) for the initial and fused auxiliary spaces:

$$
A_{0}(\lambda+i \kappa) A_{n-1}(\lambda)=1
$$

Gathering the above two equations (6.18), (6.19) we conclude

$$
A_{0}(\lambda) A_{0}(-\lambda)=1
$$

Additional constraints are obtained on the dressing functions from analyticity properties. Studying carefully the common poles of successive $g_{l}$ 's, we deduce from the form of the $g_{i}$ functions (6.14) (6.16) that $g_{l}$ and $g_{l-1}$ have common poles at $\lambda=-\frac{i l}{2}$, therefore from analyticity requirements

$$
A_{l}\left(-\frac{i l}{2}\right)=A_{l-1}\left(-\frac{i l}{2}\right), \quad l=1, \ldots, k-1 .
$$

The last relation is valid for $s o(n)$ and $s p(n)$ as well. However, there is one extra constraint specific to $s o(2 k+1)$, namely

$$
A_{k}\left(-\frac{i k}{2}\right)=A_{k-1}\left(-\frac{i k}{2}\right)
$$

Having deduced the necessary constraints for the dressing functions, we now determine them explicitly. The dressing functions $A_{l}$ are essentially characterised by a set of parameters $\left\{\lambda_{j}^{(l)} \mid j=\right.$ $\left.1, \ldots, M^{(l)}\right\}$, where the integer numbers $M^{(l)}$ are related to the eigenvalues of diagonal generators of $s o(n), s p(n)$. Defining these generators as:

$$
S^{(l)}=\sum_{i=1}^{N} s_{i}^{(l)}, \quad s^{(l)}=E_{l l}-E_{\overline{l l}} .
$$

The precise identification of $M$ follows from the symmetry of the transfer matrix (see also [31]), in particular

$$
S^{(l)}=M^{(l-1)}-M^{(l)}, \quad l=1, \ldots, k-2
$$


which is valid for $s o(2 k+1), s o(2 k)$ and $s p(2 k)$. The two remaining quantum numbers are given by

$$
\begin{array}{ll}
\text { For } s o(2 k+1): \quad S^{(k-1)}=M^{(k-2)}-M^{(k-1)} ; \quad S^{(k)}=M^{(k-1)}-M^{(k)} ; \\
\text { For } s o(2 k): & S^{(k-1)}=M^{(k-2)}-M^{(+)}-M^{(-)} ; \quad S^{(k)}=M^{(+)}-M^{(-)} ; \\
\text {For } s p(2 k): & S^{(k-1)}=M^{(k-2)}-M^{(k-1)} ; \quad S^{(k)}=M^{(k-1)}-2 M^{(k)} .
\end{array}
$$

We have also $M^{(0)}=N$ (valid for both $s p(n)$ and $s o(n)$ algebras).

Let us point out that, away from the boundaries, the behavior of the chain is considered to be as in the bulk. Therefore, the above quantum numbers describe accurately enough the states of the system. The dressing functions are given by the following expressions:

\section{A. $\operatorname{so}(2 \mathrm{k}+1)$}

$$
\begin{aligned}
A_{0}(\lambda)= & \prod_{j=1}^{M^{(1)}} \frac{\lambda+\lambda_{j}^{(1)}-\frac{i}{2}}{\lambda+\lambda_{j}^{(1)}+\frac{i}{2}} \frac{\lambda-\lambda_{j}^{(1)}-\frac{i}{2}}{\lambda-\lambda_{j}^{(1)}+\frac{i}{2}}, \\
A_{l}(\lambda)= & \prod_{j=1}^{M^{(l)}} \frac{\lambda+\lambda_{j}^{(l)}+i+\frac{i l}{2}}{\lambda+\lambda_{j}^{(l)}+\frac{i l}{2}} \frac{\lambda-\lambda_{j}^{(l)}+\frac{i l}{2}+i}{\lambda-\lambda_{j}^{(l)}+\frac{i l}{2}} \\
& \times \prod_{j=1}^{M^{(l+1)}} \frac{\lambda+\lambda_{j}^{(l+1)}+\frac{i l}{2}-\frac{i}{2}}{\lambda+\lambda_{j}^{(l+1)}+\frac{i l}{2}+\frac{i}{2}} \frac{\lambda-\lambda_{j}^{(l+1)}+\frac{i l}{2}-\frac{i}{2}}{\lambda-\lambda_{j}^{(l+1)}+\frac{i l}{2}+\frac{i}{2}}, \quad l=1, \ldots, k-1 \\
A_{k}(\lambda)= & \prod_{j=1}^{M^{(k)}} \frac{\lambda+\lambda_{j}^{(k)}+\frac{i k}{2}-i}{\lambda+\lambda_{j}^{(k)}+\frac{i k}{2}} \frac{\lambda-\lambda_{j}^{(k)}+\frac{i k}{2}-i}{\lambda-\lambda_{j}^{(k)}+\frac{i k}{2}} \frac{\lambda+\lambda_{j}^{(k)}+\frac{i k}{2}+\frac{i}{2}}{\lambda+\lambda_{j}^{(k)}+\frac{i k}{2}-\frac{i}{2}} \frac{\lambda-\lambda_{j}^{(k)}+\frac{i k}{2}+\frac{i}{2}}{\lambda-\lambda_{j}^{(k)}+\frac{i k}{2}-\frac{i}{2}}
\end{aligned}
$$

together with $A_{l}(\lambda)=A_{n-l-1}(-\lambda-i \kappa)$ for $l>k$. We recall that for $s o(2 k+1), \kappa=k-\frac{1}{2}$.

\section{B. $\operatorname{so}(2 \mathrm{k})$}

The dressing functions $A_{l}(\lambda)$ are the same as in the $s o(2 k+1)$ case for $l=0, \ldots, k-3$, while

$$
\begin{aligned}
A_{k-2}(\lambda)= & \prod_{j=1}^{M^{(k-2)}} \frac{\lambda+\lambda_{j}^{(k-2)}+\frac{i k}{2}}{\lambda+\lambda_{j}^{(k-2)}+\frac{i k}{2}-i} \frac{\lambda-\lambda_{j}^{(k-2)}+\frac{i k}{2}}{\lambda-\lambda_{j}^{(k-2)}+\frac{i k}{2}-i} \\
& \times \prod_{j=1}^{M^{(+)}} \frac{\lambda+\lambda_{j}^{(+)}+\frac{i k}{2}-\frac{3 i}{2}}{\lambda+\lambda_{j}^{(+)}+\frac{i k}{2}-\frac{i}{2}} \frac{\lambda-\lambda_{j}^{(+)}+\frac{i k}{2}-\frac{3 i}{2}}{\lambda-\lambda_{j}^{(+)}+\frac{i k}{2}-\frac{i}{2}} \\
& \times \prod_{j=1}^{M^{(-)}} \frac{\lambda+\lambda_{j}^{(-)}+\frac{i k}{2}-\frac{3 i}{2}}{\lambda+\lambda_{j}^{(-)}+\frac{i k}{2}-\frac{i}{2}} \frac{\lambda-\lambda_{j}^{(-)}+\frac{i k}{2}-\frac{3 i}{2}}{\lambda-\lambda_{j}^{(-)}+\frac{i k}{2}-\frac{i}{2}},
\end{aligned}
$$




$$
\begin{aligned}
A_{k-1}(\lambda)= & \prod_{j=1}^{M^{(+)}} \frac{\lambda+\lambda_{j}^{(+)}+\frac{i k}{2}-\frac{3 i}{2}}{\lambda+\lambda_{j}^{(+)}+\frac{i k}{2}-\frac{i}{2}} \frac{\lambda-\lambda_{j}^{(+)}+\frac{i k}{2}-\frac{3 i}{2}}{\lambda-\lambda_{j}^{(+)}+\frac{i k}{2}-\frac{i}{2}} \\
& \times \prod_{j=1}^{M^{(-)}} \frac{\lambda+\lambda_{j}^{(-)}+\frac{i k}{2}+\frac{i}{2}}{\lambda+\lambda_{j}^{(-)}+\frac{i k}{2}-\frac{i}{2}} \frac{\lambda-\lambda_{j}^{(-)}+\frac{i k}{2}+\frac{i}{2}}{\lambda-\lambda_{j}^{(-)}+\frac{i k}{2}-\frac{i}{2}}
\end{aligned}
$$

with still $A_{l}(\lambda)=A_{n-l-1}(-\lambda-i \kappa)$ for $l>k-1$, with $\kappa=k-1$.

\section{C. $\operatorname{sp}(2 k)$}

Similarly, the dressing functions are the same as in the $s o(2 k+1)$ case for $l=0, \ldots, k-3$, and:

$$
\begin{aligned}
A_{k-2}(\lambda)= & \prod_{j=1}^{M^{(k-2)}} \frac{\lambda+\lambda_{j}^{(k-2)}+\frac{i k}{2}}{\lambda+\lambda_{j}^{(k-2)}+\frac{i k}{2}-i} \frac{\lambda-\lambda_{j}^{(k-2)}+\frac{i k}{2}}{\lambda-\lambda_{j}^{(k-2)}+\frac{i k}{2}-i} \\
& \times \prod_{j=1}^{M^{(k-1)}} \frac{\lambda+\lambda_{j}^{(k-1)}+\frac{i k}{2}-\frac{3 i}{2}}{\lambda+\lambda_{j}^{(k-1)}+\frac{i k}{2}-\frac{i}{2}} \frac{\lambda_{j}^{(k-1)}+\frac{i k}{2}-\frac{3 i}{2}}{\lambda-\lambda_{j}^{(k-1)}+\frac{i k}{2}-\frac{i}{2}} \\
A_{k-1}(\lambda)= & \prod_{j=1}^{M^{(k-1)}} \frac{\lambda+\lambda_{j}^{(k-1)}+\frac{i k}{2}+\frac{i}{2}}{\lambda+\lambda_{j}^{(k-1)}+\frac{i k}{2}-\frac{i}{2}} \frac{\lambda-\lambda_{j}^{(k-1)}+\frac{i k}{2}+\frac{i}{2}}{\lambda-\lambda_{j}^{(k-1)}+\frac{i k}{2}-\frac{i}{2}} \\
& \times \prod_{j=1}^{M^{(k)}} \frac{\lambda+\lambda_{j}^{(k)}+\frac{i k}{2}-\frac{3 i}{2}}{\lambda+\lambda_{j}^{(k)}+\frac{i k}{2}+\frac{i}{2}} \frac{\lambda_{j}^{(k)}+\frac{i k}{2}-\frac{3 i}{2}}{\lambda-\lambda_{j}^{(k)}+\frac{i k}{2}+\frac{i}{2}}
\end{aligned}
$$

In addition $A_{l}(\lambda)=A_{n-l-1}(-\lambda-i \kappa)$ for $l>k-1$; note that now $\kappa=k+1$.

\subsection{Bethe Ansatz equations for $K^{-}=1$}

The above dressing functions $A_{l}$ satisfy all the imposed constraints and they are unambiguously defined.

Requiring now the analyticity of the eigenvalues, we deduce the Bethe Ansatz equations. More specifically, successive $A_{l}$ 's have common poles, which must disappear. Hence, the sum of corresponding residues of $A_{l}$ and $A_{l+1}$ in the eigenvalue expression (6.17) must be zero. The Bethe Ansatz equations immediately follow from this condition.

Let us define

$$
e_{x}(\lambda)=\frac{\lambda+\frac{i x}{2}}{\lambda-\frac{i x}{2}}
$$

for any $x$. Then, the Bethe Ansatz equations read: 


\section{A. $\operatorname{so}(2 \mathrm{k}+1)$}

$$
\begin{aligned}
e_{1}\left(\lambda_{i}^{(1)}\right)^{2 N}= & \prod_{j=1, j \neq i}^{M^{(1)}} e_{2}\left(\lambda_{i}^{(1)}-\lambda_{j}^{(1)}\right) e_{2}\left(\lambda_{i}^{(1)}+\lambda_{j}^{(1)}\right) \prod_{j=1}^{M^{(2)}} e_{-1}\left(\lambda_{i}^{(1)}-\lambda_{j}^{(2)}\right) e_{-1}\left(\lambda_{i}^{(1)}+\lambda_{j}^{(2)}\right), \\
1= & \prod_{j=1, j \neq i}^{M^{(l)}} e_{2}\left(\lambda_{i}^{(l)}-\lambda_{j}^{(l)}\right) e_{2}\left(\lambda_{i}^{(l)}+\lambda_{j}^{(l)}\right) \prod_{\eta= \pm 1}^{M^{(l+\eta)}} \prod_{j=1}^{l} e_{-1}\left(\lambda_{i}^{(l)}-\lambda_{j}^{(l+\eta)}\right) e_{-1}\left(\lambda_{i}^{(l)}+\lambda_{j}^{(l+\eta)}\right) \\
& \\
1= & \prod_{j=1, j \neq i}^{M^{(k)}} e_{1}\left(\lambda_{i}^{(k)}-\lambda_{j}^{(k)}\right) e_{1}\left(\lambda_{i}^{(k)}+\lambda_{j}^{(k)}\right) \prod_{j=1}^{M^{(k-1)}} e_{-1}\left(\lambda_{i}^{(k)}-\lambda_{j}^{(k-1)}\right) e_{-1}\left(\lambda_{i}^{(k)}+\lambda_{j}^{(k-1)}\right)
\end{aligned}
$$

\section{B. $\operatorname{so}(2 \mathrm{k})$}

The first $k-3$ equations are the same as in $s o(2 k+1)$, see eq. (6.30), but the last three equations are modified, namely

$$
\begin{aligned}
1= & \prod_{j=1, j \neq i}^{M^{(k-2)}} e_{2}\left(\lambda_{i}^{(k-2)}-\lambda_{j}^{(k-2)}\right) e_{2}\left(\lambda_{i}^{(k-2)}+\lambda_{j}^{(k-2)}\right) \prod_{j=1}^{M^{(k-3)}} e_{-1}\left(\lambda_{i}^{(k-2)}-\lambda_{j}^{(k-3)}\right) e_{-1}\left(\lambda_{i}^{(k-2)}+\lambda_{j}^{(k-3)}\right) \\
& \times \prod_{j=1}^{M^{(+)}} e_{-1}\left(\lambda_{i}^{(k-2)}-\lambda_{j}^{(+)}\right) e_{-1}\left(\lambda_{i}^{(k-2)}+\lambda_{j}^{(+)}\right) \prod_{j=1}^{M^{(-)}} e_{-1}\left(\lambda_{i}^{(k-2)}-\lambda_{j}^{(-)}\right) e_{-1}\left(\lambda_{i}^{(k-2)}+\lambda_{j}^{(-)}\right) \\
1= & \prod_{j=1, j \neq i}^{M^{(\tau)}} e_{2}\left(\lambda_{i}^{(\tau)}-\lambda_{j}^{(\tau)}\right) e_{2}\left(\lambda_{i}^{(\tau)}+\lambda_{j}^{(\tau)}\right) \prod_{j=1}^{M^{(k-2)}} e_{-1}\left(\lambda_{i}^{(\tau)}-\lambda_{j}^{(k-2)}\right) e_{-1}\left(\lambda_{i}^{(\tau)}+\lambda_{j}^{(k-2)}\right), \tau= \pm .
\end{aligned}
$$

\section{C. $\operatorname{sp}(2 \mathrm{k})$}

The first $k-2$ equations are the same as in the $s o(2 k+1)$, while the last two equations are given by

$$
\begin{aligned}
1= & \prod_{j=1, j \neq i}^{M^{(k-1)}} e_{2}\left(\lambda_{i}^{(k-1)}-\lambda_{j}^{(k-1)}\right) e_{2}\left(\lambda_{i}^{(k-1)}+\lambda_{j}^{(k-1)}\right) \prod_{j=1}^{M^{(k-2)}} e_{-1}\left(\lambda_{i}^{(k-1)}-\lambda_{j}^{(k-2)}\right) e_{-1}\left(\lambda_{i}^{(k-1)}+\lambda_{j}^{(k-2)}\right) \\
& \times \prod_{j=1}^{M^{(k)}} e_{-2}\left(\lambda_{i}^{(k-1)}-\lambda_{j}^{(k)}\right) e_{-2}\left(\lambda_{i}^{(k-1)}+\lambda_{j}^{(k)}\right) \\
1= & \prod_{j=1, j \neq i}^{M^{(k)}} e_{4}\left(\lambda_{i}^{(k)}-\lambda_{j}^{(k)}\right) e_{4}\left(\lambda_{i}^{(k)}+\lambda_{j}^{(k)}\right) \prod_{j=1}^{M^{(k-1)}} e_{-2}\left(\lambda_{i}^{(k)}-\lambda_{j}^{(k-1)}\right) e_{-2}\left(\lambda_{i}^{(k)}+\lambda_{j}^{(k-1)}\right) .
\end{aligned}
$$

For all these cases, we recover the rational limits of the equations given in [34, 35]. 


\subsection{Eigenvalues and Bethe Ansatz equations for diagonal $K^{-}$}

Until now, we have considered the case of trivial boundary effects $K^{+}=K^{-}=1$. We here come to the main point of our derivation and insert non-trivial boundary effects. We shall then rederive modified Bethe Ansatz equations. We choose $K^{-}$to be one of the diagonal solutions D1, D2, D3, D4. We consider, for simplicity but without loss of generality, $K^{+}=1$. Remark that the pseudo-vacuum remains an exact eigenstate after this modification.

Let us rewrite the solutions D1, D2, D3 and D4 in a slightly modified notation, which we are going to use from now on.

D1: The solution D1 can be written in the following form

$$
K(\lambda)=\operatorname{diag}(\alpha, \ldots, \alpha, \beta, \ldots, \beta)
$$

The number of $\alpha^{\prime} s$ is equal to the number of $\beta^{\prime} s$, so that this solution exists only for the $s o(2 k)$ and $s p(2 k)$ cases as stated in Proposition 3.1, and

$$
\alpha(\lambda)=-\lambda+i \xi, \quad \beta(\lambda)=\lambda+i \xi
$$

where $\xi=\frac{1}{c}$ is the free boundary parameter.

D2: Solution D2 can be written as

$$
K(\lambda)=\operatorname{diag}(\alpha, \beta, \ldots, \beta, \gamma)
$$

with

$$
\alpha(\lambda)=\frac{-\lambda+i \xi_{1}}{\lambda+i \xi_{1}}, \quad \beta(\lambda)=1, \quad \gamma(\lambda)=\frac{-\lambda+i \xi_{n}}{\lambda+i \xi_{n}}
$$

where $\xi_{1}=-\frac{1}{c_{1}}, \xi_{n}=-\frac{1}{c_{n}}$ are the boundary parameters which satisfy the constraint

$$
\xi_{1}+\xi_{n}=\kappa-1
$$

We remind that this solution exists for $s o(n)$ algebras, but not for $\operatorname{sp}(n)$.

D3: Solution D3 has the form

$$
K(\lambda)=\operatorname{diag}(\alpha, \ldots, \alpha, \beta, \ldots, \beta, \alpha, \ldots, \alpha) .
$$

The number of $\alpha^{\prime} s$ is $2 m$ and the number of $\beta^{\prime} s$ is $n-2 m$, where the free integer parameter $m$ obeys $1 \leq m \leq k-1$ for $s o(2 k), s p(2 k)$ and $1 \leq m \leq k$ for $s o(2 k+1)$. Moreover

$$
\alpha(\lambda)=-\lambda+i \xi, \quad \beta(\lambda)=\lambda+i \xi
$$

where $\xi=\frac{n}{4}-m$ has a fixed value, unlike the $s u(n)$ case where the corresponding solution has a free boundary parameter (see [4, 41]). 
D4: Finally solution D4, which holds only for the so(4) case, can be written in the following form

$$
K(\lambda)=\operatorname{diag}(\alpha, \beta, \gamma, \delta)
$$

where

$$
\begin{aligned}
& \alpha(\lambda)=\left(-\lambda+i \xi_{-}\right)\left(-\lambda+i \xi_{+}\right), \quad \beta(\lambda)=\left(\lambda+i \xi_{-}\right)\left(-\lambda+i \xi_{+}\right) \\
& \gamma(\lambda)=\left(-\lambda+i \xi_{-}\right)\left(\lambda+i \xi_{+}\right), \quad \delta(\lambda)=\left(\lambda+i \xi_{-}\right)\left(\lambda+i \xi_{+}\right) .
\end{aligned}
$$

$\xi_{-}=\frac{1}{c_{2}}, \xi_{+}=\frac{1}{c_{3}}$ are both free parameters.

We now come to the explicit expression of the eigenvalues when $K^{-}$is one of the above mentioned solutions. We should point out that the dressing functions are related to the bulk behavior of the chain and thus they are form-invariant under changes of boundary conditions. Indeed what is modified in the expression of the eigenvalues (6.17) are the $g_{l}$ functions which characterise the boundary effects. We call the new $g_{l}$ functions $\tilde{g}_{l}$.

D1: As already mentioned, the solution D1 can only be applied in $s o(2 k)$ and $s p(2 k)$. In this case we have

$$
\begin{aligned}
& \tilde{g}_{l}(\lambda)=(-\lambda+i \xi) g_{l}(\lambda), \quad l=0, \ldots, k-1 \\
& \tilde{g}_{l}(\lambda)=(\lambda+i \xi+i \kappa) g_{l}(\lambda), \quad l=k, \ldots, 2 k-1
\end{aligned}
$$

where $g(\lambda)$ are given by (6.14)- 6.16). The system with such boundaries has a residual symmetry $s l(k)$ in both cases, which immediately follows from the structure of the corresponding $K$ matrix.

D2: We have

$$
\begin{aligned}
& \tilde{g}_{0}(\lambda)=\frac{\left(-\lambda+i \xi_{1}\right)}{\left(\lambda+i \xi_{1}\right)} g_{0}(\lambda), \quad \tilde{g}_{n-1}(\lambda)=\frac{\left(\lambda+i \xi_{1}+i\right)}{\left(\lambda+i \xi_{1}\right)} \frac{\left(\lambda+i \xi_{1}+i \kappa\right)}{\left(-\lambda-i \kappa+i \xi_{1}+i\right)} g_{n-1}(\lambda), \\
& \tilde{g}_{l}(\lambda)=\frac{\left(\lambda+i \xi_{1}+i\right)}{\left(\lambda+i \xi_{1}\right)} g_{l}(\lambda), \quad l=1, \ldots, n-2
\end{aligned}
$$

Again the $g_{l}(\lambda)$ are given by (6.14) -6.16). Similarly, from the structure of the $K$ matrix we conclude that the residual symmetry is $s o(n-2) \otimes s o(2)$.

D3: For the D3 solution we find the following modified $g$ functions

$$
\begin{aligned}
& \tilde{g}_{l}(\lambda)=(-\lambda+i \xi) g_{l}(\lambda), \quad l=0, \ldots, m-1 \\
& \tilde{g}_{l}(\lambda)=\left(\lambda+\frac{i \kappa}{2} \pm \frac{i}{2}\right) g_{l}(\lambda), \quad l=m, \ldots, n-m-1 \\
& \tilde{g}_{l}(\lambda)=(-\lambda-i \kappa-i \xi) \frac{\left(\lambda+\frac{i \kappa}{2} \pm \frac{i}{2}\right)}{\left(\lambda+\frac{i \kappa}{2} \mp \frac{i}{2}\right)} g_{l}(\lambda), \quad l=n-m, \ldots, n-1 .
\end{aligned}
$$

The symmetry of the transfer matrix for this $K$ matrix is $s o(n-2 m) \otimes s o(2 m)$, (resp. $s p(n-2 m) \otimes$ $\operatorname{sp}(2 m))$. 
D4: Finally for solution D4 the modified $g$ functions are given by

$$
\begin{array}{ll}
\tilde{g}_{0}(\lambda)=\left(-\lambda+i \xi_{-}\right)\left(-\lambda+i \xi_{+}\right) g_{0}(\lambda), & \tilde{g}_{1}(\lambda)=\left(\lambda+i \xi_{-}+i\right)\left(-\lambda+i \xi_{+}\right) g_{1}(\lambda) \\
\tilde{g}_{2}(\lambda)=\left(\lambda+i \xi_{+}+i\right)\left(-\lambda+i \xi_{-}\right) g_{2}(\lambda), & \tilde{g}_{3}(\lambda)=\left(\lambda+i \xi_{-}+i\right)\left(\lambda+i \xi_{+}+i\right) g_{3}(\lambda) .
\end{array}
$$

We now formulate the Bethe Ansatz equations for the general diagonal solutions. The only modifications induced on equations (6.30)-(6.32) are the following for each solution:

D1 The factor $-e_{2 \xi+\kappa}^{-1}(\lambda)$ appears in the LHS of the $k^{\text {th }}$ Bethe equation.

D2 The factor $-e_{2 \xi_{1}+1}^{-1}(\lambda)$ appears in the LHS of the first Bethe equation.

D3 The factor $-e_{2 \xi+m}^{-1}(\lambda)$ appears in the LHS of the $m^{\text {th }}$ Bethe equation with $m=1, \ldots, k-1$ for $s o(2 k+1), s p(2 k)$ and $m=1, \ldots, k-2$ for $s o(2 k)$. For $s o(2 k)$, when $m=k-1$, the factor $-e_{1}^{-1}(\lambda)$ appears in the LHS of the $(k-1)^{t h}$ and $k^{\text {th }}$ Bethe Ansatz equations.

We treat solution D4 separately in the next section.

\section{$7 \quad$ Ground state and excitations}

The next step is to determine the ground state and the low-lying excitations of the model. One of the main aims of this work is indeed the computation of the scattering of the low-lying excitations off the boundaries. The bulk scattering for these models has been already studied in [27, nevertheless we are going to rederive these results as a check in the next section.

We recall that the quantum numbers that describe a state are given by (6.23), and the energy is derived via the relation $H=\left.\frac{d}{d \lambda} t(\lambda)\right|_{\lambda=0}$. It is given by

$$
E=-\frac{1}{2 \pi} \sum_{j=1}^{M^{(1)}} \frac{1}{\left(\lambda_{j}^{(1)}\right)^{2}+\frac{1}{4}}
$$

In what follows we write the Bethe Ansatz equations for the ground state and the low-lying excitations (holes) of the models under study. Bethe Ansatz equations may in general only be solved in the thermodynamic limit $N \rightarrow \infty$. In this limit, it is assumed that a state is described in particular by the density functions $\sigma^{l}(\lambda)$ of the parameters $\lambda_{i}^{(l)}$.

We here make the hypothesis that non trivial boundary effects do not modify the nature of the ground state and excited states but only the values of the Bethe parameters.

\section{A. $\operatorname{so}(2 \mathrm{k}+1)$}

Let us first consider the $s o(2 k+1)$ case, for which the ground state consists of $k-1$ filled Dirac seas, whereas the $k^{\text {th }}$ sea is filled with two-strings of the form $\lambda_{0} \pm \frac{i}{4}$. Remark the shift $\frac{i}{4}$, instead of $\frac{i}{2}$ usually expected, because the length of the $k^{t h}$ root in the $s o(2 k+1)$ case is half the length of the other roots (see also [27]). This leads us to rewrite the Bethe Ansatz equations for the ground state 
and the low-lying excitations (holes) in the following form, inserting the two-strings contribution in the $k^{\text {th }}$ set:

$$
\begin{aligned}
& e_{1}\left(\lambda_{i}^{(1)}\right)^{2 N+1}=-\prod_{j=1}^{M^{(1)}} e_{2}\left(\lambda_{i}^{(1)}-\lambda_{j}^{(1)}\right) e_{2}\left(\lambda_{i}^{(1)}+\lambda_{j}^{(1)}\right) \prod_{j=1}^{M^{(2)}} e_{-1}\left(\lambda_{i}^{(1)}-\lambda_{j}^{(2)}\right) e_{-1}\left(\lambda_{i}^{(1)}+\lambda_{j}^{(2)}\right), \\
& e_{1}\left(\lambda_{i}^{(l)}\right)=-\prod_{j=1}^{M^{(l)}} e_{2}\left(\lambda_{i}^{(l)}-\lambda_{j}^{(l)}\right) e_{2}\left(\lambda_{i}^{(l)}+\lambda_{j}^{(l)}\right) \prod_{\tau= \pm 1} \prod_{j=1}^{M^{(l+\tau)}} e_{-1}\left(\lambda_{i}^{(l)}-\lambda_{j}^{(l+\tau)}\right) e_{-1}\left(\lambda_{i}^{(l)}+\lambda_{j}^{(l+\tau)}\right) \\
& (l=2, \ldots, k-2) \\
& e_{1}\left(\lambda_{i}^{(k-1)}\right)=-\prod_{j=1}^{M^{(k-1)}} e_{2}\left(\lambda_{i}^{(k-1)}-\lambda_{j}^{(k-1)}\right) e_{2}\left(\lambda_{i}^{(k-1)}+\lambda_{j}^{(k-1)}\right) \\
& \times \prod_{j=1}^{M^{(k-2)}} e_{-1}\left(\lambda_{i}^{(k-1)}-\lambda_{j}^{(k-2)}\right) e_{-1}\left(\lambda_{i}^{(k-1)}+\lambda_{j}^{(k-2)}\right) \\
& \times \prod_{j=1}^{M^{(k)}} e_{-\frac{1}{2}}\left(\lambda_{i}^{(k-1)}-\lambda_{j}^{(k)}\right) e_{-\frac{1}{2}}\left(\lambda_{i}^{(k-1)}+\lambda_{j}^{(k)}\right) e_{-\frac{3}{2}}\left(\lambda_{i}^{(k-1)}-\lambda_{j}^{(k)}\right) e_{-\frac{3}{2}}\left(\lambda_{i}^{(k-1)}+\lambda_{j}^{(k)}\right) \\
& e_{1}\left(\lambda_{i}^{(k)}\right)=-\prod_{j=1}^{M^{(k)}} e_{1}\left(\lambda_{i}^{(k)}-\lambda_{j}^{(k)}\right)^{2} e_{1}\left(\lambda_{i}^{(k)}+\lambda_{j}^{(k)}\right)^{2} e_{2}\left(\lambda_{i}^{(k)}-\lambda_{j}^{(k)}\right) e_{2}\left(\lambda_{i}^{(k)}+\lambda_{j}^{(k)}\right) \\
& \times \prod_{j=1}^{M^{(k-1)}} e_{-\frac{1}{2}}\left(\lambda_{i}^{(k)}-\lambda_{j}^{(k-1)}\right) e_{-\frac{1}{2}}\left(\lambda_{i}^{(k)}+\lambda_{j}^{(k-1)}\right) e_{-\frac{3}{2}}\left(\lambda_{i}^{(k)}-\lambda_{j}^{(k-1)}\right) e_{-\frac{3}{2}}\left(\lambda_{i}^{(k)}+\lambda_{j}^{(k-1)}\right)
\end{aligned}
$$

For the general diagonal solutions of the reflection equation we have to multiply:

- for D2, the LHS of the $1^{\text {st }}$ Bethe Ansatz equation with $-e_{2 \xi_{1}+1}^{-1}$;

- for D3, we multiply the LHS of the $m^{\text {th }}$ Bethe Ansatz equation with $-e_{2 \xi+m}^{-1}, 1 \leq m \leq k-1$.

We are interested in the low-lying excitations, which are holes in the filled sea and are highest weight representations of $s o(2 k+1)$. We restrict ourselves here to the states with $\nu^{(l)}$ holes in the $l$ sea, which correspond to the vector representations of $s o(2 k+1)$, and also to holes in the $k$ sea, which corresponds to the $2^{k}$-dimensional spinor representation (see also [31]). We convert the sums into integrals by employing the following approximate relation 42 .

$$
\frac{1}{N} \sum_{i=1}^{M} f\left(\lambda_{i}^{(l)}\right)=\int_{0}^{\infty} d \lambda f(\lambda) \sigma^{l}(\lambda)-\frac{1}{N} \sum_{i=1}^{\nu^{(l)}} f\left(\tilde{\lambda}_{i}^{(l)}\right)-\frac{1}{2 N} f(0)+O\left(\frac{1}{N^{2}}\right)
$$

where the correction terms take into account the $\nu^{(l)}$ holes located at values $\tilde{\lambda}_{i}^{(l)}$ and the contribution at $0^{+}$. We shall denote by $\hat{f}(\omega)$ the Fourier transform of any function $f(\lambda)$.

Once we take the logarithm and the derivative of (17.2), we extract the densities from the equation

$$
\hat{\mathcal{K}}(\omega) \hat{\sigma}(\omega)=\hat{a}(\omega)+\frac{1}{N} \hat{F}(\omega)+\frac{1}{N} \hat{G}(\omega, \xi)
$$


where $a_{x}(\lambda)=\frac{i}{2 \pi} \frac{d}{d \lambda} \ln e_{x}(\lambda)$ and $\hat{a}_{x}(\omega)=e^{-\frac{x \omega}{2}}$. We have introduced

$$
a(\lambda)=\left(\begin{array}{c}
2 a_{1}(\lambda) \\
0 \\
\vdots \\
0
\end{array}\right), \quad \sigma(\lambda)=\left(\begin{array}{c}
\sigma^{1}(\lambda) \\
\vdots \\
\sigma^{l}(\lambda) \\
\vdots \\
\sigma^{k}(\lambda)
\end{array}\right)
$$

$F(\lambda), G(\lambda, \xi)$ are $k$ component vectors as well with

$$
\begin{aligned}
F^{j}(\lambda) & =a_{1}(\lambda) \delta_{j 1}-a_{1}(\lambda)+a_{2}(\lambda)+\sum_{j=1}^{\nu^{(l)}}\left(a_{2}\left(\lambda-\tilde{\lambda}_{j}^{(l)}\right)+a_{2}\left(\lambda+\tilde{\lambda}_{j}^{(l)}\right)\right) \delta_{l j} \\
& -\sum_{j=1}^{\nu^{(l)}}\left(a_{1}\left(\lambda-\tilde{\lambda}_{j}^{(l)}\right)+a_{1}\left(\lambda+\tilde{\lambda}_{j}^{(l)}\right)\right)\left(\delta_{j, l+1}+\delta_{j, l-1}\right), \quad(j=1, \ldots, k-2) \\
F^{k-1}(\lambda)= & a_{2}(\lambda)-\left(a_{\frac{1}{2}}(\lambda)+a_{\frac{3}{2}}(\lambda)\right)-\sum_{j=1}^{\nu^{(l)}}\left(a_{1}\left(\lambda-\tilde{\lambda}_{j}^{(l)}\right)+a_{1}\left(\lambda+\tilde{\lambda}_{j}^{(l)}\right)\right) \delta_{l, k-2} \\
& +\sum_{j=1}^{\nu^{(l)}}\left(a_{2}\left(\lambda-\tilde{\lambda}_{j}^{(l)}\right)+a_{2}\left(\lambda+\tilde{\lambda}_{j}^{(l)}\right)\right) \delta_{l, k-1}-\sum_{j=1}^{\nu^{(l)}}\left(\left(a_{\frac{1}{2}}+a_{\frac{3}{2}}\right)\left(\lambda-\tilde{\lambda}_{j}^{(l)}\right)+\left(a_{\frac{1}{2}}+a_{\frac{3}{2}}\right)\left(\lambda+\tilde{\lambda}_{j}^{(l)}\right)\right) \delta_{k l} \\
F^{k}(\lambda)= & 3 a_{1}(\lambda)+a_{2}(\lambda)-\left(a_{\frac{1}{2}}(\lambda)+a_{\frac{3}{2}}(\lambda)\right)-\sum_{j=1}^{\nu^{(l)}}\left(\left(a_{\frac{1}{2}}+a_{\frac{3}{2}}\right)\left(\lambda-\tilde{\lambda}_{j}^{(l)}\right)+\left(a_{\frac{1}{2}}+a_{\frac{3}{2}}\right)\left(\lambda+\tilde{\lambda}_{j}^{(l)}\right)\right) \delta_{k-1, l} \\
& +\sum_{j=1}^{\nu^{(l)}}\left(\left(2 a_{1}+a_{2}\right)\left(\lambda-\tilde{\lambda}_{j}^{(l)}\right)+\left(2 a_{1}+a_{2}\right)\left(\lambda+\tilde{\lambda}_{j}^{(l)}\right)\right) \delta_{k l}
\end{aligned}
$$

and the $\xi$ dependent part is

$$
\begin{array}{ll}
\text { for D2: } & G^{j}(\lambda, \xi)=-a_{2 \xi_{1}+1}(\lambda) \delta_{j 1}, \\
\text { for D3: } & G^{j}(\lambda, \xi)=-a_{2 \xi+m}(\lambda) \delta_{j m} \quad(m=1, \ldots, k-1) .
\end{array}
$$

Finally, the non vanishing entries of $\hat{\mathcal{K}}$ are

$$
\begin{aligned}
\hat{\mathcal{K}}_{i j}(\omega) & =\left(1+\hat{a}_{2}(\omega)\right) \delta_{i j}-\hat{a}_{1}(\omega)\left(\delta_{i, j+1}+\delta_{i, j-1}\right), \quad i, j=1, \ldots, k-1 \\
\hat{\mathcal{K}}_{k-1, k}(\omega) & =\hat{\mathcal{K}}_{k, k-1}(\omega)=-\left(\hat{a}_{\frac{1}{2}}(\omega)+\hat{a}_{\frac{3}{2}}(\omega)\right), \\
\hat{\mathcal{K}}_{k k}(\omega) & =1+2 \hat{a}_{1}(\omega)+\hat{a}_{2}(\omega) .
\end{aligned}
$$

The solution of (17.4) has the form

$$
\sigma(\lambda)=2 \epsilon(\lambda)+\frac{1}{N} \Phi_{0}(\lambda)+\frac{1}{N} \Phi_{1}(\lambda, \xi)
$$


where $\epsilon$ and $\Phi_{0,1}$ are $k$ component vectors with

$$
\hat{\epsilon}^{i}(\omega)=\hat{\mathcal{R}}_{i 1}(\omega) \hat{a}_{1}(\omega), \quad \hat{\Phi}_{0}^{i}(\omega)=\sum_{j=1}^{k} \hat{\mathcal{R}}_{i j}(\omega) \hat{F}^{j}(\omega), \quad \hat{\Phi}_{1}^{i}(\omega, \xi)=\sum_{j=1}^{k} \hat{\mathcal{R}}_{i j}(\omega) \hat{G}^{j}(\omega, \xi)
$$

$\hat{\mathcal{R}}=\hat{\mathcal{K}}^{-1}$ and $\hat{\epsilon}^{j}$ is the energy of a hole in the $j$ sea which can be written in terms of hyperbolic functions

$$
\begin{aligned}
& \hat{\epsilon}^{j}(\omega)=\frac{\cosh \left(k-\frac{1}{2}-j\right) \frac{\omega}{2}}{\cosh \left(k-\frac{1}{2}\right) \frac{\omega}{2}}, \quad j=1, \ldots, k-1, \quad \hat{\epsilon}^{k}(\omega)=\frac{1}{2 \cosh \left(k-\frac{1}{2}\right) \frac{\omega}{2}} \\
& \hat{\mathcal{R}}_{i j}(\omega)=e^{\frac{\omega}{2}} \frac{\sinh \min (i, j) \frac{\omega}{2} \cosh \left(k-\frac{1}{2}-\max (i, j)\right) \frac{\omega}{2}}{\cosh \left(k-\frac{1}{2}\right) \frac{\omega}{2} \sinh \frac{\omega}{2}}, \quad i, j=1, \ldots, k-1 \\
& \hat{\mathcal{R}}_{j k}(\omega)=\hat{\mathcal{R}}_{k j}(\omega)=\frac{e^{\frac{\omega}{2}}}{2} \frac{\sinh \frac{j \omega}{2}}{\cosh \left(k-\frac{1}{2}\right) \frac{\omega}{2} \sinh \frac{\omega}{2}}, j=1, \ldots, k-1 \\
& \hat{\mathcal{R}}_{k k}(\omega)=\frac{e^{\frac{\omega}{2}}}{2} \frac{\sinh \frac{k \omega}{2}}{2 \cosh \frac{\omega}{4} \cosh \left(k-\frac{1}{2}\right) \frac{\omega}{2} \sinh \frac{\omega}{2}}
\end{aligned}
$$

\section{B. $\operatorname{so}(2 \mathrm{k})$}

In this case, the ground state consists of $k$ filled Dirac seas of real strings. Therefore the Bethe Ansatz equations have exactly the same form as in (6.31). For the general diagonal solutions of the reflection equation, we have to multiply:

- for D1, the LHS of the $k^{\text {th }}$ Bethe Ansatz equation with $-e_{2 \xi+\kappa}^{-1}$,

- for D2, the LHS of the $1^{\text {st }}$ equation with $-e_{2 \xi_{1}+1}^{-1}$,

- for D3, the LHS of the $m^{\text {th }}$ equation with $-e_{2 \xi+m}^{-1}$ for $m=1, \ldots, k-2$, while the LHS of the $(k-1)^{t h}$ and $k^{t h}$ equations for $m=k-1$ are multiplied by $-e_{1}^{-1}$.

In this case as well, we restrict ourselves to states with $\nu^{(l)}$ holes in the $l$ sea. Note that now the spinor representation splits into two spinor representations of dimension $2^{k-1}$ (see also 31]), and the holes in the,+- sea correspond exactly to these two spinor representations. The densities satisfy the same equation (7.4) as in the $s o(2 k+1)$ case with $\sigma$ and $a$ given by (7.5) and

$$
\hat{\epsilon}^{j}(\omega)=\frac{\cosh (k-1-j) \frac{\omega}{2}}{\cosh (k-1) \frac{\omega}{2}}, \quad j=1, \ldots, k-2 \text { and } \hat{\epsilon}^{ \pm}(\omega)=\frac{1}{2 \cosh (k-1) \frac{\omega}{2}}
$$




$$
\begin{aligned}
F^{j}(\lambda) & =a_{1}(\lambda) \delta_{j 1}-a_{1}(\lambda)+a_{2}(\lambda)+\sum_{j=1}^{\nu^{(l)}}\left(a_{2}\left(\lambda-\tilde{\lambda}_{j}^{(l)}\right)+a_{2}\left(\lambda+\tilde{\lambda}_{j}^{(l)}\right)\right) \delta_{l j} \\
& -\sum_{j=1}^{\nu^{(l)}}\left(a_{1}\left(\lambda-\tilde{\lambda}_{j}^{(l)}\right)+a_{1}\left(\lambda+\tilde{\lambda}_{j}^{(l)}\right)\right)\left(\delta_{j, l+1}+\delta_{j, l-1}\right), \quad(j=1, \ldots, k-3) \\
F^{k-2}(\lambda)= & a_{2}(\lambda)-2 a_{1}(\lambda)+\sum_{j=1}^{\nu^{(l)}}\left(a_{2}\left(\lambda-\tilde{\lambda}_{j}^{(l)}\right)+a_{2}\left(\lambda+\tilde{\lambda}_{j}^{(l)}\right)\right) \delta_{l, k-2} \\
& -\sum_{j=1}^{\nu^{(l)}}\left(a_{1}\left(\lambda-\tilde{\lambda}_{j}^{(l)}\right)+a_{1}\left(\lambda+\tilde{\lambda}_{j}^{(l)}\right)\right)\left(\delta_{l, k-3}+\delta_{l+}+\delta_{l-}\right) \\
F^{ \pm}(\lambda) & =a_{2}(\lambda)+\sum_{j=1}^{\nu^{(l)}}\left(a_{2}\left(\lambda-\tilde{\lambda}_{j}^{(l)}\right)+a_{2}\left(\lambda+\tilde{\lambda}_{j}^{(l)}\right)\right) \delta_{l \pm}-\sum_{j=1}^{\nu^{(l)}}\left(a_{1}\left(\lambda-\tilde{\lambda}_{j}^{(l)}\right)+a_{1}\left(\lambda+\tilde{\lambda}_{j}^{(l)}\right)\right) \delta_{l, k-2}
\end{aligned}
$$

- for D1: $\quad G^{j}(\lambda, \xi)=-a_{2 \xi+\kappa}(\lambda) \delta_{j k}$,

- for D2: $\quad G^{j}(\lambda, \xi)=-a_{\left(2 \xi_{1}+1\right)}(\lambda) \delta_{j 1}$,

- for D3: $\quad\left\{\begin{array}{l}G^{j}(\lambda, \xi)=-a_{(2 \xi+m)}(\lambda) \delta_{j m} \quad(m=1, \ldots, k-2), \\ G^{i}(\lambda)=-a_{1}(\lambda)\left(\delta_{i, k-1}+\delta_{i, k}\right) \quad(m=k-1) .\end{array}\right.$

Again, the non-vanishing entries of $\hat{\mathcal{K}}$ are

$$
\begin{aligned}
& \hat{\mathcal{K}}_{i j}(\omega)=\left(1+\hat{a}_{2}(\omega)\right) \delta_{i j}-\hat{a}_{1}(\omega)\left(\delta_{i, j+1}+\delta_{i, j-1}\right), i, j=1, \ldots, k-2, \\
& \hat{\mathcal{K}}_{k-2, \pm}(\omega)=\hat{\mathcal{K}}_{ \pm, k-2}(\omega)=-\hat{a}_{1}(\omega), \\
& \hat{\mathcal{K}}_{--}(\omega)=\hat{\mathcal{K}}_{++}(\omega)=1+\hat{a}_{2}(\omega) \text { and } \hat{\mathcal{K}}_{+-}(\omega)=\hat{\mathcal{K}}_{-+}(\omega)=0 .
\end{aligned}
$$

We solve equation (7.4) and find the densities $\sigma^{i}$ which describe a Bethe Ansatz state. The solution of (17.4) has the same form as in (17.9) with

$$
\begin{aligned}
& \hat{\mathcal{R}}_{i j}(\omega)=e^{\frac{\omega}{2}} \frac{\sinh \min (i, j) \frac{\omega}{2} \cosh (k-1-\max (i, j)) \frac{\omega}{2}}{\cosh (k-1) \frac{\omega}{2} \sinh \frac{\omega}{2}}, i, j=1, \ldots, k-2, \\
& \hat{\mathcal{R}}_{j \pm}(\omega)=\hat{\mathcal{R}}_{ \pm j}(\omega)=\frac{e^{\frac{\omega}{2}}}{2} \frac{\sinh \frac{j \omega}{2}}{\cosh (k-1) \frac{\omega}{2} \sinh \frac{\omega}{2}}, j=1, \ldots, k-2 \\
& \hat{\mathcal{R}}_{++}(\omega)=\hat{\mathcal{R}}_{--}(\omega)=\frac{e^{\frac{\omega}{2}}}{2} \frac{\sinh \frac{k \omega}{2}}{2 \cosh \frac{\omega}{2} \cosh (k-1) \frac{\omega}{2} \sinh \frac{\omega}{2}} \\
& \hat{\mathcal{R}}_{+-}(\omega)=\hat{\mathcal{R}}_{-+}(\omega)=\frac{e^{\frac{\omega}{2}}}{2} \frac{\sinh (k-2) \frac{\omega}{2}}{2 \cosh \frac{\omega}{2} \cosh (k-1) \frac{\omega}{2} \sinh \frac{\omega}{2}}
\end{aligned}
$$

Let us now consider the particular solution D4 for the so(4) case. The corresponding Bethe Ansatz equations, as in the bulk, are basically two copies of the $X X X$ spin chain equations $(s o(4)=$ 
$s u(2) \otimes s u(2))$, namely

$$
e_{2 \xi_{\tau}+1}\left(\lambda_{i}^{(\tau)}\right)^{-1} e_{1}\left(\lambda_{i}^{(\tau)}\right)^{2 N+1}=\prod_{j=1}^{M^{(\tau)}} e_{2}\left(\lambda_{i}^{(\tau)}-\lambda_{j}^{(\tau)}\right) e_{2}\left(\lambda_{i}^{(\tau)}+\lambda_{j}^{(\tau)}\right)
$$

where $\tau= \pm$. It is obvious that the only representations that remain are the two two-dimensional

spinor representations. The Bethe Ansatz equations are then two decoupled equations, as it is also evident from (7.12): one has $\mathcal{R}_{+-}(\omega)=\mathcal{R}_{-+}(\omega)=0$; moreover $\mathcal{R}_{++}(\omega), \mathcal{R}_{--}(\omega)$ are given by (17.17) for $k=2$, and

$$
F^{\tau}(\lambda)=a_{1}(\lambda)+a_{2}(\lambda)+\sum_{j=1}^{\nu^{(\tau)}}\left(a_{2}\left(\lambda-\tilde{\lambda}_{j}^{(\tau)}\right)+a_{2}\left(\lambda+\tilde{\lambda}_{j}^{(\tau)}\right)\right), \quad G^{\tau}\left(\lambda, \xi_{\tau}\right)=-a_{2 \xi_{\tau}+1}(\lambda)
$$

\section{C. $\operatorname{sp}(2 \mathrm{k})$}

The ground state in this case consists of $k-1$ filled Dirac seas of two-strings $\left(\lambda_{0}^{(j)} \pm \frac{i}{2}\right)$, and the $k$ sea is filled with real strings. The Bethe Ansatz equations take the form

$$
\begin{aligned}
e_{2}\left(\lambda_{i}^{(1)}\right)^{2 N+1}= & -\prod_{j=1}^{M^{(1)}} e_{2}^{2}\left(\lambda_{i}^{(1)}-\lambda_{j}^{(1)}\right) e_{2}^{2}\left(\lambda_{i}^{(1)}+\lambda_{j}^{(1)}\right) e_{4}\left(\lambda_{i}^{(1)}-\lambda_{j}^{(1)}\right) e_{4}\left(\lambda_{i}^{(1)}+\lambda_{j}^{(1)}\right) \\
& \times \prod_{j=1}^{M^{(2)}} e_{-1}\left(\lambda_{i}^{(1)}-\lambda_{j}^{(2)}\right) e_{-1}\left(\lambda_{i}^{(1)}+\lambda_{j}^{(2)}\right) e_{-3}\left(\lambda_{i}^{(1)}-\lambda_{j}^{(2)}\right) e_{-3}\left(\lambda_{i}^{(1)}+\lambda_{j}^{(2)}\right), \\
e_{2}\left(\lambda_{i}^{(l)}\right)= & -\prod_{i j=1}^{M^{(l)}} e_{2}^{2}\left(\lambda_{i}^{(l)}-\lambda_{j}^{(l)}\right) e_{2}^{2}\left(\lambda_{i}^{(l)}+\lambda_{j}^{(l)}\right) e_{4}\left(\lambda_{i}^{(l)}-\lambda_{j}^{(l)}\right) e_{4}\left(\lambda_{i}^{(l)}+\lambda_{j}^{(l)}\right) \\
& \times \prod_{\tau= \pm 1} \prod_{j=1}^{M^{(l+\tau)}} e_{-1}\left(\lambda_{i}^{(l)}-\lambda_{j}^{(l+\tau)}\right) e_{-1}\left(\lambda_{i}^{(l)}+\lambda_{j}^{(l+\tau)}\right) e_{-3}\left(\lambda_{i}^{(l)}-\lambda_{j}^{(l+\tau)}\right) e_{-3}\left(\lambda_{i}^{(l)}+\lambda_{j}^{(l+\tau)}\right) \\
& l=2, \ldots, k-1 \\
e_{2}\left(\lambda_{i}^{(k)}\right)= & -\prod_{j=1}^{M^{(k)}} e_{4}\left(\lambda_{i}^{(k)}-\lambda_{j}^{(k)}\right) e_{4}\left(\lambda_{i}^{(k)}+\lambda_{j}^{(k)}\right) \\
& \times \prod_{j=1}^{M^{(k-1)}} e_{-1}\left(\lambda_{i}^{(k)}-\lambda_{j}^{(k-1)}\right) e_{-1}\left(\lambda_{i}^{(k)}+\lambda_{j}^{(k-1)}\right) e_{-3}\left(\lambda_{i}^{(k)}-\lambda_{j}^{(k-1)}\right) e_{-3}\left(\lambda_{i}^{(k)}+\lambda_{j}^{(k-1)}\right)
\end{aligned}
$$

where for the general diagonal solutions of the reflection equation we have to multiply:

- for D1, the LHS of the $k^{\text {th }}$ equation with $-e_{2 \xi+\kappa}^{-1}$,

- for D3, the LHS of the $m^{t h}$ equations with $e_{2 \xi+m+1}^{-1} e_{2 \xi+m-1}^{-1}, m=1, \ldots, k-1$. 
Again the densities for the state with $\nu^{(l)}$ holes in the $l$ sea satisfy the same equations (17.4) as in the $s o(2 k+1)$ case with $\sigma$ given by (17.5),$a^{j}(\lambda)=2 a_{2}(\lambda) \delta_{j 1}$ and

$$
\begin{aligned}
\hat{\epsilon}^{j}(\omega)=\frac{\cosh (k+1-j) \frac{\omega}{2}}{2 \cosh \frac{\omega}{2} \cosh (k+1) \frac{\omega}{2}}, \quad j=1, \ldots, k \\
F^{j}(\lambda)=3 a_{2}(\lambda)+a_{4}(\lambda)-2\left(a_{1}(\lambda)+a_{3}(\lambda)\right)+\left(a_{1}(\lambda)+a_{3}(\lambda)\right) \delta_{j 1} \\
+\sum_{j=1}^{\nu^{(l)}}\left(\left(2 a_{2}+a_{4}\right)\left(\lambda-\tilde{\lambda}_{j}^{(l)}\right)+\left(2 a_{2}+a_{4}\right)\left(\lambda+\tilde{\lambda}_{j}^{(l)}\right)\right) \delta_{j l} \\
-\sum_{j=1}^{\nu^{(l)}}\left(\left(a_{1}+a_{3}\right)\left(\lambda-\tilde{\lambda}_{j}^{(l)}\right)+\left(a_{1}+a_{3}\right)\left(\lambda+\tilde{\lambda}_{j}^{(l)}\right)\right)\left(\delta_{j, l+1}+\delta_{j, l-1}\right), \\
F^{k}(\lambda)=a_{2}(\lambda)+a_{4}(\lambda)-\left(a_{1}(\lambda)+a_{3}(\lambda)\right)+\sum_{j=1}^{\nu^{(l)}}\left(a_{4}\left(\lambda-\tilde{\lambda}_{j}^{(l)}\right)+a_{4}\left(\lambda+\tilde{\lambda}_{j}^{(l)}\right)\right) \delta_{l k} \\
-\sum_{j=1}^{\nu^{(l)}}\left(\left(a_{1}+a_{3}\right)\left(\lambda-\tilde{\lambda}_{j}^{(l)}\right)+\left(a_{1}+a_{3}\right)\left(\lambda+\tilde{\lambda}_{j}^{(l)}\right)\right) \delta_{l, k-1},
\end{aligned}
$$

$$
\begin{array}{ll}
\text { for D1: } & G^{j}(\lambda, \xi)=-a_{2 \xi+\kappa}(\lambda) \delta_{j k} \\
\text { for D3: } & G^{j}(\lambda, \xi)=-\left(a_{2 \xi+m+1}(\lambda)+a_{2 \xi+m-1}(\lambda)\right) \delta_{j m} \quad(m=1, \ldots, k-1)
\end{array}
$$

The non-vanishing entries of $\hat{\mathcal{K}}$ are

$$
\begin{aligned}
\hat{\mathcal{K}}_{i j}(\omega) & =\left(1+\hat{a}_{2}(\omega)\right)^{2} \delta_{i j}-\left(\hat{a}_{1}(\omega)+\hat{a}_{3}(\omega)\right)\left(\delta_{i, j+1}+\delta_{i, j-1}\right), \quad i, j=1, \ldots, k-1 \\
\hat{\mathcal{K}}_{k-1, k}(\omega) & =\hat{\mathcal{K}}_{k, k-1}(\omega)=-\left(\hat{a}_{1}(\omega)+\hat{a}_{3}(\omega)\right), \\
\hat{\mathcal{K}}_{k k}(\omega) & =1+\hat{a}_{4}(\omega) .
\end{aligned}
$$

As in the previous cases the solution of (7.4) has the form (7.9), (7.10) with

$$
\hat{\mathcal{R}}_{i j}(\omega)=\frac{e^{\omega}}{2 \cosh \frac{\omega}{2}} \frac{\sinh \min (i, j) \frac{\omega}{2} \cosh (k+1-\max (i, j)) \frac{\omega}{2}}{\cosh (k+1) \frac{\omega}{2} \sinh \frac{\omega}{2}}, \quad i, j=1, \ldots, k
$$

\section{Scattering}

Having obtained the excitations of the model, we are ready to compute the complete boundary $S$ matrix. For this purpose we follow the formulation developed by Korepin, and later by Andrei and Destri [43, 44]. First we have to implement the so-called quantisation condition,

$$
\left(e^{2 i N p^{l}} S-1\right)\left|\tilde{\lambda}_{i}^{(l)}\right\rangle=0
$$


where $p^{l}$ is the momentum of the particle (in our case, the hole) with rapidity $\tilde{\lambda}_{1}^{l}$. For the case of $\nu$ (even) holes in $l$ sea we insert the integrated density (7.9) into the quantisation condition (8.1). We use the dispersion relation

$$
\epsilon^{l}(\lambda)=\frac{1}{2 \pi} \frac{d}{d \lambda} p^{l}(\lambda)
$$

and the sum rule $N \int_{0}^{\tilde{\lambda}_{i}} d \lambda \sigma(\lambda) \in \mathbb{Z}_{+}$. The boundary $S$ matrix for the right boundary is taken to be one of the diagonal solutions D1, D2, D3, D4, whereas the boundary $S$ matrix for the left boundary is proportional to unit. We end up with the following expression for the boundary scattering amplitudes:

$$
\alpha^{+l} \alpha^{-l}=\exp \left\{2 \pi N \int_{0}^{\tilde{\lambda}_{1}} d \lambda\left(\sigma^{l}(\lambda)-2 \epsilon^{l}(\lambda)\right)\right\}
$$

with

$$
\alpha^{-l}(\lambda, \xi)=k_{0}(\lambda) k_{1}(\lambda, \xi), \quad \alpha^{+l}(\lambda)=k_{0}(\lambda)
$$

where $\alpha^{-l}$ is the first element of the diagonal boundary $S$ matrix. It is obvious that $\alpha^{+l}$ has no $\xi$ dependence and realises just the overall factor in front of the unit matrix at the left boundary (recall that $\left.K^{+}=1\right)$. Moreover,

$$
k_{0}\left(\tilde{\lambda}_{1}^{l}\right)=\exp \left\{i \pi \int_{0}^{\tilde{\lambda}_{1}^{l}} d \lambda \Phi_{0}^{l}(\lambda)\right\}, \quad k_{1}\left(\tilde{\lambda}_{1}^{l}, \xi\right)=\exp \left\{2 i \pi \int_{0}^{\tilde{\lambda}_{1}^{l}} d \lambda \Phi_{1}^{l}\left(\tilde{\lambda}_{1}^{l}, \xi\right)\right\}
$$

with $\Phi_{0,1}^{l}$ given by (7.10). We finally restrict ourselves to $l=1$ in the first sea and we write the latter expression in term of the Fourier transform (7.10) of $\Phi_{0,1}^{1}$,

$$
k_{0}(\lambda)=\exp \left\{-\frac{1}{2} \int_{-\infty}^{\infty} \frac{d \omega}{\omega} \hat{\Phi}_{0}^{1}(\omega) e^{-i \omega \lambda}\right\}, \quad k_{1}(\lambda, \xi)=\exp \left\{-\int_{-\infty}^{\infty} \frac{d \omega}{\omega} \hat{\Phi}_{1}^{1}(\omega, \xi) e^{-i \omega \lambda}\right\} .
$$

In what follows we express the scattering amplitudes in terms of $\Gamma$-functions.

\section{A. $\operatorname{so}(\mathbf{n})$}

Before we write down the explicit expressions for the boundary $S$ matrices, let us recall the form of the exact bulk $S$ matrix. It is easy to compute the scattering amplitude between two holes (vectors) in the first sea. The bulk scattering amplitude comes from the contribution of the terms of $\Phi_{0}^{1}$ given by eqs. (17.6) (17.10), (7.12), (7.14), with argument $\lambda \pm \tilde{\lambda}_{j}$. After some algebra and using the following identity

$$
\frac{1}{2} \int_{0}^{\infty} \frac{d \omega}{\omega} \frac{e^{-\frac{\mu \omega}{2}}}{\cosh \frac{\omega}{2}}=\ln \frac{\Gamma\left(\frac{\mu+1}{4}\right)}{\Gamma\left(\frac{\mu+3}{4}\right)}
$$

we conclude that the hole-hole scattering amplitude is given by the expression

$$
S_{0}(\lambda)=\frac{\tan \pi\left(\frac{i \lambda-1}{n-2}\right)}{\tan \pi\left(\frac{i \lambda+1}{n-2}\right)} \frac{\Gamma\left(\frac{i \lambda}{n-2}\right)}{\Gamma\left(\frac{-i \lambda}{n-2}\right)} \frac{\Gamma\left(\frac{-i \lambda}{n-2}+\frac{1}{2}\right)}{\Gamma\left(\frac{i \lambda}{n-2}+\frac{1}{2}\right)} \frac{\Gamma\left(\frac{-i \lambda+1}{n-2}\right)}{\Gamma\left(\frac{i \lambda+1}{n-2}\right)} \frac{\Gamma\left(\frac{i \lambda+1}{n-2}+\frac{1}{2}\right)}{\Gamma\left(\frac{-i \lambda+1}{n-2}+\frac{1}{2}\right)} .
$$


The explicit bulk $S$ matrix then has the following structure ${ }^{3}$

$$
S(\lambda)=\frac{S_{0}(\lambda)}{(i \lambda+\kappa)(i \lambda+1)}(i \lambda(i \lambda+\kappa) \mathbb{I}+(i \lambda+\kappa) P-i \lambda Q)
$$

Now, we give the expressions for the boundary $S$ matrix, which follow from (8.5), (8.6), and the duplication formula for the $\Gamma$ function

$$
2^{2 x-1} \Gamma\left(x+\frac{1}{2}\right) \Gamma(x)=\pi^{\frac{1}{2}} \Gamma(2 x) .
$$

The $\xi$-independent part of the overall factor $k_{0}$, eq. (8.5), is given by

$$
k_{0}(\lambda)=Y_{0}(\lambda) \frac{\Gamma\left(\frac{i \lambda}{n-2}\right)}{\Gamma\left(\frac{-i \lambda}{n-2}\right)} \frac{\Gamma\left(\frac{-i \lambda}{n-2}+\frac{3}{4}\right)}{\Gamma\left(\frac{i \lambda}{n-2}+\frac{3}{4}\right)} \frac{\Gamma\left(\frac{i \lambda+1 / 2}{n-2}+\frac{3}{4}\right)}{\Gamma\left(\frac{-i \lambda+1 / 2}{n-2}+\frac{3}{4}\right)} \frac{\Gamma\left(\frac{-i \lambda+1 / 2}{n-2}+\frac{1}{2}\right)}{\Gamma\left(\frac{i \lambda+1 / 2}{n-2}+\frac{1}{2}\right)}
$$

where

$$
Y_{0}(\lambda)=\frac{\sin \pi\left(\frac{i \lambda+1 / 2}{n-2}-\frac{1}{4}\right)}{\sin \pi\left(\frac{i \lambda-1 / 2}{n-2}+\frac{1}{4}\right)} \frac{\sin \pi\left(\frac{i \lambda-1 / 2}{n-2}+\frac{1}{2}\right)}{\sin \pi\left(\frac{i \lambda+1 / 2}{n-2}-\frac{1}{2}\right)} \frac{\sin \pi\left(\frac{i \lambda}{n-2}+\frac{1}{4}\right)}{\sin \pi\left(\frac{i \lambda}{n-2}-\frac{1}{4}\right)} .
$$

Note that our solution includes the necessary CDD factors both for the bulk and boundary matrices (see also [21, 27]). The expression for the $\xi$-dependant part $k_{1}$ depends on which solutions D1, D2, D3, D4 we consider:

\section{D1:}

$$
k_{1}\left(\lambda, \xi^{\prime}\right)=\frac{\Gamma\left(\frac{i \lambda+\xi^{\prime}}{n-2}+\frac{1}{2}\right)}{\Gamma\left(\frac{-i \lambda+\xi^{\prime}}{n-2}+\frac{1}{2}\right)} \frac{\Gamma\left(\frac{-i \lambda+\xi^{\prime}}{n-2}+1\right)}{\Gamma\left(\frac{\xi^{\prime}+i \lambda}{n-2}+1\right)}
$$

where $\xi^{\prime}=\xi-\frac{1}{2}$ is the renormalised boundary parameter (see also [41]). We also compute the $\beta$ element of the $K$ matrix (6.33) by employing the "duality" transformation $\xi \rightarrow-\xi$, and the symmetry of the $K$ matrix and of the transfer matrix (see also [41). In particular, we obtain a set of Bethe Ansatz equations for $\xi \rightarrow-\xi$, which allows to determine the difference

$$
\hat{\Phi}_{1}^{1}(\omega,-\xi)-\hat{\Phi}_{1}^{1}(\omega, \xi)=e^{-(2 \xi-1) \frac{\omega}{2}}
$$

and consequently

$$
\frac{\beta^{-}(\lambda, \xi)}{\alpha^{-}(\lambda, \xi)}=e_{2 \xi^{\prime}}(\lambda)
$$

This provides us with a consistency check for our procedure since one obtains independently the exact ratio between different elements of the reflection matrix. Thus, we have completely determined the $K$ matrix that corresponds to the solution D1.

\footnotetext{
${ }^{3}$ We do not compute all the eigenvalues of the bulk $S$ matrix via the Bethe Ansatz. Such a general computation, in the bulk, is beyond the scope of this work.
} 
D2:

$$
k_{1}\left(\lambda, \xi^{\prime}\right)=\frac{\tan \pi\left(\frac{i \lambda-\xi_{n}^{\prime}}{n-2}\right)}{\tan \pi\left(\frac{i \lambda+\xi_{n}^{\prime}}{n-2}\right)} \frac{\Gamma\left(\frac{i \lambda+\xi_{1}^{\prime}}{n-2}+\frac{1}{2}\right)}{\Gamma\left(\frac{-i \lambda+\xi_{1}^{\prime}}{n-2}+\frac{1}{2}\right)} \frac{\Gamma\left(\frac{-i \lambda+\xi_{1}^{\prime}}{n-2}+1\right)}{\Gamma\left(\frac{i \lambda+\xi_{1}^{\prime}}{n-2}+1\right)} \frac{\Gamma\left(\frac{i \lambda+\xi_{n}^{\prime}}{n-2}+\frac{1}{2}\right)}{\Gamma\left(\frac{-i \lambda+\xi_{n}^{\prime}}{n-2}+\frac{1}{2}\right)} \frac{\Gamma\left(\frac{-i \lambda+\xi_{n}^{\prime}}{n-2}\right)}{\Gamma\left(\frac{i \lambda+\xi_{n}^{\prime}}{n-2}\right)}
$$

where $\xi_{1}^{\prime}=\xi_{1}-\frac{1}{2}$ and $\xi_{n}^{\prime}=\xi_{n}+\frac{1}{2}$ are the renormalised boundary parameters. Remark that the constraint $\xi_{1}+\xi_{n}=\kappa-1$ is also true for the renormalised boundary parameters, namely $\xi_{1}^{\prime}+\xi_{n}^{\prime}=\kappa-1$. Similarly we employ the duality transformation which, for this solution, reads $\xi_{1} \rightarrow \xi_{n}$. We then determine the difference

$$
\hat{\Phi}_{1}^{1}\left(\omega, \xi_{n}, \xi_{1}\right)-\hat{\Phi}_{1}^{1}\left(\omega, \xi_{1}, \xi_{n}\right)=e^{-\left(2 \xi_{1}-1\right) \frac{\omega}{2}}+e^{\left(2 \xi_{n}+1\right) \frac{\omega}{2}}
$$

and the ratio

$$
\frac{\gamma^{-}\left(\lambda, \xi_{1}, \xi_{n}\right)}{\alpha^{-}\left(\lambda, \xi_{1}, \xi_{n}\right)}=e_{2 \xi_{1}^{\prime}}(\lambda) e_{-2 \xi_{n}^{\prime}}(\lambda)
$$

Again we have here an independent consistency check.

Of course, we need to determine one further ratio, namely $\frac{\beta^{-}\left(\lambda, \xi_{1}, \xi_{n}\right)}{\alpha^{-}\left(\lambda, \xi_{1}, \xi_{n}\right)}$. We have not been able to explicitly extract this information from the Bethe Ansatz formulation. Nevertheless, since the $K$ matrix is a solution of the reflection equation, the only choice we have for this ratio is

$$
\frac{\beta^{-}\left(\lambda, \xi_{1}, \xi_{n}\right)}{\alpha^{-}\left(\lambda, \xi_{1}, \xi_{n}\right)}=e_{2 \xi_{1}^{\prime}}(\lambda)
$$

With this, we have completed the derivation of the $K$ matrix that corresponds to the solution D2.

D3: The $\xi$-dependent part of the $K$ matrix overall factor that corresponds to D3 is

$$
k_{1}\left(\lambda, \xi^{\prime}\right)=\frac{\Gamma\left(\frac{i \lambda+\xi^{\prime}}{n-2}+\frac{1}{2}\right)}{\Gamma\left(\frac{-i \lambda+\xi^{\prime}}{n-2}+\frac{1}{2}\right)} \frac{\Gamma\left(\frac{-i \lambda+\xi^{\prime}}{n-2}+1\right)}{\Gamma\left(\frac{i \lambda+\xi^{\prime}}{n-2}+1\right)} \frac{\Gamma\left(\frac{-i \lambda+1 / 2}{n-2}+\frac{3}{4}\right)}{\Gamma\left(\frac{i \lambda+1 / 2}{n-2}+\frac{3}{4}\right)} \frac{\Gamma\left(\frac{i \lambda+1 / 2}{n-2}+\frac{1}{4}\right)}{\Gamma\left(\frac{-i \lambda+1 / 2}{n-2}+\frac{1}{4}\right)} .
$$

The total overall factor for this solution is

$$
\begin{aligned}
k_{0}(\lambda) k_{1}\left(\lambda, \xi^{\prime}\right)= & Y_{1}(\lambda) \frac{\Gamma\left(\frac{i \lambda}{n-2}\right)}{\Gamma\left(\frac{-i \lambda}{n-2}\right)} \frac{\Gamma\left(\frac{-i \lambda}{n-2}+\frac{3}{4}\right)}{\Gamma\left(\frac{i \lambda}{n-2}+\frac{3}{4}\right)} \frac{\Gamma\left(\frac{i \lambda+1 / 2}{n-2}+\frac{1}{4}\right)}{\Gamma\left(\frac{-i \lambda+1 / 2}{n-2}+\frac{1}{4}\right)} \frac{\Gamma\left(\frac{-i \lambda+1 / 2}{n-2}+\frac{1}{2}\right)}{\Gamma\left(\frac{i \lambda+1 / 2}{n-2}+\frac{1}{2}\right)} \\
& \times \frac{\Gamma\left(\frac{i \lambda+\xi^{\prime}}{n-2}+\frac{1}{2}\right)}{\Gamma\left(\frac{-i \lambda+\xi^{\prime}}{n-2}+\frac{1}{2}\right)} \frac{\Gamma\left(\frac{-i \lambda+\xi^{\prime}}{n-2}+1\right)}{\Gamma\left(\frac{i \lambda+\xi^{\prime}}{n-2}+1\right)}
\end{aligned}
$$

where

$$
Y_{1}(\lambda)=\frac{\sin \pi\left(\frac{i \lambda+1 / 2}{n-2}-\frac{1}{4}\right)}{\sin \pi\left(\frac{i \lambda-1 / 2}{n-2}+\frac{1}{4}\right)} \frac{\sin \pi\left(\frac{i \lambda-1 / 2}{n-2}+\frac{1}{2}\right)}{\sin \pi\left(\frac{i \lambda+1 / 2}{n-2}-\frac{1}{2}\right)} \frac{\sin \pi\left(\frac{i \lambda}{n-2}+\frac{1}{4}\right)}{\sin \pi\left(\frac{i \lambda}{n-2}-\frac{1}{4}\right)} .
$$

The latter expression agrees with the one found in [21], although we find slightly different CDD factors. Again $\xi^{\prime}=\xi-\frac{1}{2}$ is the renormalised boundary parameter which now must have the fixed 
value $\xi^{\prime}=\frac{n}{4}-m$, to ensure the resulting $K$ matrix satisfies the reflection equation. Unfortunately, in this case, we cannot employ a duality transformation to derive the ratio $\frac{\beta^{-}}{\alpha^{-}}$(6.38). However, since the $K$ matrix is a solution of the reflection equation the ratio must be

$$
\frac{\beta^{-}(\lambda)}{\alpha^{-}(\lambda)}=e_{2 \xi^{\prime}}(\lambda)
$$

D4: For this solution the $\xi$-independent part is given by (8.11) with $n=4$, while the $\xi$-dependent part is given by $(\tau= \pm)$ :

$$
k_{1 \tau}\left(\lambda, \xi_{\tau}^{\prime}\right)=\frac{\Gamma\left(\frac{i \lambda+\xi_{\tau}^{\prime}}{2}+\frac{1}{4}\right)}{\Gamma\left(\frac{-i \lambda+\xi_{\tau}^{\prime}}{2}+\frac{1}{4}\right)} \frac{\Gamma\left(\frac{-i \lambda+\xi_{\tau}^{\prime}}{2}+\frac{3}{4}\right)}{\Gamma\left(\frac{i \lambda+\xi_{\tau}^{\prime}}{2}+\frac{3}{4}\right)} .
$$

We exploit the duality transformation one more time, $\xi_{\tau} \rightarrow-\xi_{\tau}$, to calculate the ratio

$$
\frac{\beta_{\tau}^{-}(\lambda)}{\alpha_{\tau}^{-}(\lambda)}=e_{2 \xi_{\tau}^{\prime}}(\lambda)
$$

with the renormalised boundary parameter $\xi_{\tau}^{\prime}=\xi_{\tau}-\frac{1}{2}$. We recall that the two spinor representations are two-dimensional, and the corresponding $K$ matrices are of course two-dimensional with

$$
K_{\tau}^{-}\left(\lambda, \xi_{\tau^{\prime}}\right)=\operatorname{diag}\left(\alpha_{\tau}^{-}\left(\lambda, \xi_{\tau}^{\prime}\right), \beta_{\tau}^{-}\left(\lambda, \xi_{\tau}^{\prime}\right)\right)
$$

In other words, we have obtained two copies of the $X X X$ boundary $S$ matrix, with two free boundary parameters $\xi_{\tau}^{\prime}$.

\section{B. $\operatorname{sp}(\mathbf{n})$}

The corresponding bulk scattering amplitude for the $s p(n)$ is given by the following expression

$$
S_{0}(\lambda)=\frac{\tan \pi\left(\frac{i \lambda-1}{n+2}\right)}{\tan \pi\left(\frac{i \lambda+1}{n+2}\right)} \frac{\Gamma\left(\frac{i \lambda}{n+2}\right)}{\Gamma\left(\frac{-i \lambda}{n+2}\right)} \frac{\Gamma\left(\frac{-i \lambda}{n+2}+\frac{1}{2}\right)}{\Gamma\left(\frac{i \lambda}{n+2}+\frac{1}{2}\right)} \frac{\Gamma\left(\frac{-i \lambda+1}{n+2}\right)}{\Gamma\left(\frac{i \lambda+1}{n+2}\right)} \frac{\Gamma\left(\frac{i \lambda+1}{n+2}+\frac{1}{2}\right)}{\Gamma\left(\frac{-i \lambda+1}{n+2}+\frac{1}{2}\right)} S_{b}(\lambda)
$$

where

$$
S_{b}(\lambda)=\exp \left[-\int_{-\infty}^{\infty} \frac{d \omega}{\omega} \frac{\cosh \frac{k \omega}{2} e^{-i \omega \lambda}}{2 \cosh \frac{\omega}{2} \cosh \frac{(k+1) \omega}{2}}\right]
$$

Again the structure of the $S$ matrix is given by (8.9) with $S_{0}$ given above. $k_{0}(\lambda)$ is again given by the integral representation (8.6) using (7.10), (7.22) and (7.25). The corresponding $\xi$-dependent parts for each solution are given respectively by:

\section{D1:}

$$
k_{1}\left(\lambda, \xi^{\prime}\right)=\frac{\Gamma\left(\frac{i \lambda+\xi^{\prime}}{n+2}+\frac{1}{2}\right)}{\Gamma\left(\frac{-i \lambda+\xi^{\prime}}{n+2}+\frac{1}{2}\right)} \frac{\Gamma\left(\frac{-i \lambda+\xi^{\prime}}{n+2}+1\right)}{\Gamma\left(\frac{i \lambda+\xi^{\prime}}{n+2}+1\right)}
$$


where $\xi^{\prime}=\xi-1$ is the renormalised boundary parameter. We also compute the $\beta$ element of the $K$ matrix by employing the duality transformation $(\xi \rightarrow-\xi)$ and we obtain the difference

$$
\hat{\Phi}_{1}^{1}(\omega,-\xi)-\hat{\Phi}_{1}^{1}(\omega, \xi)=e^{-(2 \xi-2) \frac{\omega}{2}}
$$

and consequently

$$
\frac{\beta^{-}(\lambda, \xi)}{\alpha^{-}(\lambda, \xi)}=e_{2 \xi^{\prime}}(\lambda)
$$

\section{D3:}

$$
k_{1}\left(\lambda, \xi^{\prime}\right)=\frac{\tan \pi\left(\frac{i \lambda-1 / 2}{n+2}-\frac{1}{4}\right)}{\tan \pi\left(\frac{i \lambda+1 / 2}{n+2}+\frac{1}{4}\right)} \frac{\Gamma\left(\frac{i \lambda+\xi^{\prime}}{n+2}+\frac{1}{2}\right)}{\Gamma\left(\frac{-i \lambda+\xi^{\prime}}{n+2}+\frac{1}{2}\right)} \frac{\Gamma\left(\frac{-i \lambda+\xi^{\prime}}{n+2}+1\right)}{\Gamma\left(\frac{i \lambda+\xi^{\prime}}{n+2}+1\right)} \frac{\Gamma\left(\frac{-i \lambda+1 / 2}{n+2}+\frac{1}{4}\right)}{\Gamma\left(\frac{i \lambda+1 / 2}{n+2}+\frac{1}{4}\right)} \frac{\Gamma\left(\frac{i \lambda+1 / 2}{n+2}+\frac{3}{4}\right)}{\Gamma\left(\frac{-i \lambda+1 / 2}{n+2}+\frac{3}{4}\right)} .
$$

$\xi^{\prime}=\xi-1$ is the renormalised boundary parameter which must have the fixed value $\xi^{\prime}=\frac{n}{4}-m$, in order for the resulting $K$ matrix to satisfy the reflection equation. This result also agrees with the $\xi$-dependent part of the corresponding result of [21]. Unfortunately we cannot employ a duality symmetry in this case to derive the ratio $\frac{\beta}{\alpha}(6.38)$, but again, since the $K$ matrix is a solution of the reflection equation, the ratio must be

$$
\frac{\beta^{-}(\lambda)}{\alpha^{-}(\lambda)}=e_{2 \xi^{\prime}}(\lambda)
$$

\section{Conclusion}

We have established here the classification of diagonal, antidiagonal and mixed solutions to the reflection equations based on the Yangian $R$ matrices for Lie (super)algebras $s o(m), s p(n)$ and $o s p(m \mid n)$. The next step would then be to obtain the complete classification of $K$ matrices, still associated with one-dimensional quantum boundary space, which is technically more complicated. The cases of higher dimensional bulk representations, as well as operator valued $K$-matrices remain to be classified.

Some of the solutions we obtained have then been used to compute the spectrum and scattering datas for integrable spin chain systems with non trivial boundary conditions. Only the Lie algebra case has been considered here; the case of super Lie algebras $\operatorname{osp}(m \mid n)$ can now be envisioned: indeed, a suitable redefinition of indices allows us to consider a similar exact ferromagnetic pseudo-vacuum (see [45]), providing us with the starting point for the analytical Bethe Ansatz.

Remark also that the analytical Bethe Ansatz construction proposed here remains valid for upper triangular reflection matrices. Such matrices can for instance be obtained by conjugating our diagonal solutions by triangular matrices (following lemma 2.4).

In addition, the use of the analytical Bethe Ansatz method precluded applications to non-diagonal reflection matrices, for which no exact pseudo-vacuum state is available. However, this situation can be approached using the methods developed e.g. in [46, 47]. For an alternative approach, see [48]. 
Acknowledgements: We warmfully thank E. Sokatchev for discussions on symmetric spaces. This work was supported by the TMR Network "EUCLID. Integrable models and applications: from strings to condensed matter", contract number HPRN-CT-2002-00325. J.A. wishes to thank LAPTH for kind hospitality.

\section{References}

[1] I.V. Cherednik, Factorizing particles on a half line and root systems, Theor. Math. Phys. 61 (1984) 977.

[2] E.K. Sklyanin, Boundary conditions for integrable quantum systems, J. Phys. A21 (1988) 2375.

[3] S. Ghoshal and A.B. Zamolodchikov, Boundary $S$ matrix and boundary state in two-dimensional integrable quantum field theory, Int. Journ. Mod. Phys. A9 (1994) 3841 and hep-th/9306002.

[4] H.J. de Vega and A. González-Ruiz, Boundary $K$ matrices for the six vertex and the $N(2 N-1)$ $A(N-1)$ vertex models, J. Phys. A26 (1993) L519 and hep-th/9211114.

[5] A. B. Zamolodchikov and A. B. Zamolodchikov, Factorized S-matrices in two dimensions as the exact solutions of certain relativistic quantum field theory models, Annals Phys.120 (1979) 253; L. D. Faddeev, Quantum completely integrable models in field theory, Sov. Sci. Rev. C1 (1980) 107.

[6] A. Liguori, M. Mintchev and L. Zhao, Boundary exchange algebras and scattering on the halfline, Commun. Math. Phys.194 (1998) 569 and hep-th/9607085.

[7] J. Donin and A.I. Mudrov, Reflection equation, twist, and equivariant quantization, math.QA/0204295.

[8] J. Donin, P.P. Kulish and A.I. Mudrov, On universal solution to reflection equation, math.QA/0210242.

[9] P. Baseilhac and K. Koizumi, Sine-Gordon quantum field theory on the half line with quantum boundary degrees of freedom, Nucl. Phys. B649 (2003) 491 and hep-th/0208005.

[10] G.W. Delius and N.J. MacKay, Quantum group symmetry in sine-Gordon and affine Toda field theories on the half-line, Commun. Math. Phys. 233 (2003) 173 and hep-th/0112023.

[11] G.W. Delius and R.I. Nepomechie, Solutions of the boundary Yang-Baxter equation for arbitrary spin, J. Phys. A35 (2002) L341 and hep-th/0204076.

[12] R.I. Nepomechie, Boundary quantum group generators of type A, Lett. Math. Phys. 62 (2002) 83 and hep-th/0204181.

[13] J. Abad and M. Rios, Nondiagonal solutions to reflection equations in $S U(N)$ spin chains, Phys. Lett. B352 (1995) 92 and hep-th/9502129. 
[14] L. Mezincescu and R.I. Nepomechie, Fractional-spin integrals of motion for the boundary Sine-Gordon model at the free fermion point, Int. J. Mod. Phys. A13 (1998) 2747 and hep-th/9709078.

[15] A. Lima-Santos, Reflection K Matrices for 19-vertex models, Nucl. Phys. B558 (1999) 637 and solv-int/9906003.

[16] G.M. Gandenberger, New non-diagonal solutions to the $a_{n}^{(1)}$ boundary Yang-Baxter equation, hep-th/9911178.

[17] J.D. Kim, Boundary K matrix for the quantum Mikhailov-Shabat model, hep-th/9412192.

[18] V.G. Drinfel'd, Hopf algebras and the quantum Yang-Baxter equation, Soviet. Math. Dokl. 32 (1985) 254 and A new realization of Yangians and quantized affine algebras, Soviet. Math. Dokl. 36 (1988) 212.

[19] D. Arnaudon, J. Avan, N. Crampé, L. Frappat and É. Ragoucy, $R$ matrix presentation for (super)-Yangians $Y(g)$, J. Math. Phys. 44 (2003) 302 and math.QA/0111325.

[20] M. Mintchev, É. Ragoucy and P. Sorba, Spontaneous symmetry breaking in the gl(N)-NLS hierarchy on the half line, J. Phys. A34 (2001) 8345 and hep-th/0104079.

[21] N.J. MacKay and B.J. Short, Boundary scattering, symmetric spaces and the principal chiral model on the half-line, Commun. Math. Phys. 233 (2003) 313 and hep-th/0104212.

[22] M. Moriconi, Integrable boundary conditions and reflection matrices for the $O(N)$ nonlinear sigma model, Nucl. Phys. B619 (2001) 396 and hep-th/0108039.

[23] G.I. Olshanski, Twisted Yangians and infinite dimensional Lie algebras, in "Quantum groups", Lecture Notes in Math. 1510 (P. Kulish ed.), pp. 104, NY 1992.

[24] A. Molev, M. Nazarov and G. Olshanski, Yangians and classical Lie algebras, Russ. Math. Surveys 51 (1996) 205 and hep-th/9409025.

[25] M. Noumi, Macdonald's symmetric polynomials as zonal spherical functions on some quantum homogeneous spaces, Adv. Math. 123 (1996) 16.

[26] A. Molev, É. Ragoucy and P. Sorba, Coideal subalgebras in quantum affine algebras, math.QA/0208140, Rev. Math. Phys, to appear.

[27] E. Ogievetsky, N.Yu. Reshetikhin and P. Wiegmann, The principal chiral field in two-dimensions on classical Lie algebras: the Bethe Ansatz solution and factorized theory of scattering, Nucl. Phys. B280 (1987) 45.

[28] M.T. Batchelor, V. Fridkin, A. Kuniba and Y.K. Zhou, Solutions of the reflection equation for face and vertex models associated with $A_{n}^{(1)}, B_{n}^{(1)}, C_{n}^{(1)}, D_{n}^{(1)}$ and $A_{n}^{(2)}$, Phys. Lett. B376 (1996) 266 and hep-th/9601051. 
[29] M. Zirnbauer, Riemannian symmetric superspaces and their origin in random-matrix theory, J. Math. Phys. 37 (1996) 4986 and math-ph/9808012.

[30] P.P. Kulish and E.K. Sklyanin, Solutions of the Yang-Baxter equation, Zap. Nauchn. Sem. LOMI, 95 (1980) 129 and J. Sov. Math. 19 (1982) 1596.

[31] V.I. Vichirko and N.Yu. Reshetikhin, Excitation spectrum of the anisotropic generalization of an $S_{3}$ magnet, Theor. Math. Phys. 56 (1983) 805;

N.Yu. Reshetikhin, A method of functional equations in the theory of exactly solvable quantum systems, Lett. Math. Phys. 7 (1983) 205; Integrable models of quantum one-dimensional magnets with $O(n)$ and $S p(2 k)$ symmetry, Theor. Math. Phys. 63 (1985) 555; The spectrum of the transfer matrices connected with Kac-Moody algebras, Lett. Math. Phys. 14 (1987) 235.

[32] L. Mezincescu and R.I. Nepomechie, Analytical Bethe Ansatz for quantum algebra invariant spin chains, Nucl. Phys. B372 (1992) 597.

[33] A. Kuniba and J. Suzuki, Analytic Bethe Ansatz for fundamental representations of Yangians, Commun. Math. Phys. 173 (1995) 225 and hep-th/9406180.

[34] S. Artz, L. Mezincescu and R.I. Nepomechie, Spectrum of transfer matrix for $U_{q}\left(B_{n}\right)$ invariant $A_{2 n}^{(2)}$ open spin chains, Int. J. Mod. Phys. A10 (1995) 1937, and hep-th/9409130.

[35] S. Artz, L. Mezincescu and R.I. Nepomechie, Analytical Bethe Ansatz for $A_{2 n-1}^{(2)}, B_{n}^{(1)}, C_{n}^{(1)}, D_{n}^{(1)}$ quantum algebra invariant open spin chains, J. Phys. A28 (1995) 5131 and hep-th/9504085.

[36] A. Doikou, Fusion and analytical Bethe Ansatz for the $A_{n-1}^{(1)}$ open spin chain, J. Phys. A33 (2000) 4755; Quantum spin chain with "soliton nonpreserving" boundary conditions, J. Phys. A33 (2000) 8797.

[37] R.J. Baxter, Partition function of the eight-vertex lattice model, Ann. Phys. 70 (1972) 193; J. Stat. Phys. 8 (1973) 25; Exactly solved models in statistical mechanics (Academic Press, 1982)

[38] V.E. Korepin, New effects in the massive Thirring model: repulsive case, Comm. Math. Phys. 76 (1980) 165; V.E. Korepin, G. Izergin and N.M. Bogoliubov, Quantum inverse scattering method, correlation functions and algebraic Bethe Ansatz (Cambridge University Press, 1993).

[39] L. Mezincescu and R.I. Nepomechie, Fusion procedure for open chains, J. Phys. A25 (1992) 2533.

[40] Y-K. Zhou, Row transfer matrix functional relations for Baxter's eight-vertex and six-vertex models with open boundaries via more general reflection matrices, Nucl. Phys. B458 (1996) 504 , hep-th/9510095.

[41] A. Doikou and R.I. Nepomechie, Bulk and boundary $S$ matrices for the su(N) chain, Nucl. Phys. B521 (1998) 547 and hep-th/9803118; Duality and quantum algebra symmetry of 
the $A_{n-1}^{(1)}$ open spin chain with diagonal boundary fields, Nucl. Phys. B530 (1998) 641 and hep-th/9807065.

[42] M.T. Grisaru, L. Mezincescu, R.I. Nepomechie, Direct Calculation of the Boundary S Matrix for the Open Heisenberg Chain, J. Phys. A28 (1995) 1027.

[43] V. Korepin, Direct calculation of the $S$ matrix in the massive Thirring model, Theor. Math. Phys. 41 (1979) 953.

[44] N. Andrei and C. Destri, Dynamical symmetry breaking and fractionization in a new integrable model, Nucl. Phys. B231 (1984) 445.

[45] M.J. Martins, Bethe Ansatz solution of the $O s p(1 \mid 2 n)$ invariant spin chain, Phys. Lett. B359 (1995) 334.

[46] V.V. Bazhanov and N.Yu. Reshetikhin, Critical RSOS models and conformal field theory, Int. J. Mod. Phys. A4 (1989) 115.

[47] R.I. Nepomechie, Functional relations and Bethe Ansatz for the $X X Z$ chain, hep-th/0211001.

[48] J.-P. Cao, H.-Q. Lin, K.-J. Shi and Y. Wang, Exact solutions and elementary excitations in the XXZ spin chain with unparallel boundary fields, cond-mat/0212163. 